\title{
Phenomenologic aspects of seismicity in Latium
}

\author{
M. Alessio * - P. F. Biagi * \\ Received on October 15th, 1978
}

\begin{abstract}
A catalogue of the earthquakes with maximum macroseismic intensities $I_{0} \geq$ VI M.C.S. occurred in Latium during the last 20 centuries has been compiled. The study of the listed earthquakes has allowed the evidentiation of some phenomenologic aspects of Latium seismic activity and the correlation of their characteristic parameters. In particular, the study of activity migration has made it possible to indicate approximately some areas of particular interest for future research in the field of earthquakes prediction.
\end{abstract}

\section{RIASSUNTO}

Sono stati catalogati i terremoti di intensità epicentrale $I_{0}>$ VI M.C.S. che hanno interessato il Lazio dall'anno O dell'Era Volgare ai giorni nostri. L'esame dei terremoti catalogati ha consentito di evidenziare al-

$\left.{ }^{*}\right)$ Centro Interdisciplinare per le Datazioni con il ${ }^{4} \mathrm{C}$ e Istituto di Fisica dell'Università di Roma.

(***) Centro Interdisciplinare per le Datazioni con il ${ }^{14} \mathrm{C}$ e Istituto di Gcologia e Paleontologia dell'Università di Roma. 
cuni aspetti fenomenologici della sismicità laziale e di correlarne i parametri caratteristici. In particolare lo studio dell'evoluzione temporale dell'attività sismica ha reso possibile l'approssimativa indicazione di alcune aree di particolare interesse per future ricerche nel campo della previsione dei terremoti.

1. - This study deals with some of the phenomenologic aspects of seismicity in Latium, such as the focal depth, the $b$ value, the migration of activity, ignoring seismotectonic implications.

A general analysis, not taking into account the geological details of the region, allows the identification of five rather well distinct zones: proceeding from North to South, along the western belt, one: meets the true volcanic area extending from Bolsena to the Alban Hills, subdivided into two parts by the geothermal area of Cesano; to the South of the Alban Hills there are the ramifications of Valle Latina characterized by older volcanites. Moving along the western belt, one finds geological structures which are typical of Umbria and Marche in the North, and of Latium and Abruzzi in the South.

In order to carry on a detailed study of seismicity in Latium, it is convenient to examine its phenomenologic aspects in each of the five above mentioned zones, also trying to connect the various parameters characterizing the events. To this purpose the earthquakes with maximum macroseismic intensity $I_{0}>$ VI M.C.S. occurred during the last 20 centuries were taken into consideration. They area listed in Table 5 .

Information concerning the events was drawn from chronicles of the time (Agamennone, 1911-1933; Console et al., 1972; Di Filippo, 1942; Gasparini et al., 1973; Marcelli et al., 1962-1963), from old and recent national catalogues (Baratta, 1901; Caloi, 1942; Carozzo et al., 1973; Cavasino, 1935; De Panfilis, 1959), from seismological bulletins (B.C.I.S.; Bollettino I.N.G.; U.S.C.G.S.) and a critical comparison was made to derive the data appearing in the table.

2. - The serial numbers in the catalogue correspond to the different earthquakes or to the localized sequences when 
the available information was not sufficient to specify the time sequence.

For each event its date is specified and the zone including the epicentre is indicated, the symbols $1 \mathrm{~N}, 1 \mathrm{C}, 1 \mathrm{~S}, 2 \mathrm{~N}, 2 \mathrm{~S}$ corresponding to the five zones described in paragraph 1 and shown in Fig. 1 *.

Indications have been given concerning the epicentre location of the events, reporting, when possible, their coordinates as derived from microseismic data or calculated by other authors; in the other cases the localization of the epicentre presents difficulties which are greater for older events. Nevertheless, the epicentre location was evaluated using macroseismic indications found in the literature.

For intensities Mercalli-Cancani-Sieberg scale degrees are used; when an evaluation in units of some scale was not available an effort was made to make quantitative, as uniformly as possible, the qualitative information. The seismic sequences for which it was impossible to find an evaluation in term of independent events were considered as a group of seisms; their number was reckoned according to the duration of the sequence and their intensities by comparison with that of the principal shock.

When possible, the focal depth was evaluated. The values indicated with an asterisk were derived from microseismic data. When the seismograms allowed the determination of the magnitude but not of the depth, this value, indicated with $M_{k}$, was calculated using the formula (Kärník, 1969)

$$
M=\frac{2}{3} I_{\mathrm{o}}+1.4 \log h-1.25
$$

* For the denomination $1 \mathrm{~S}_{\mathrm{L}}$ of the zone relative to some earthquakes, sce paragraph 3. 


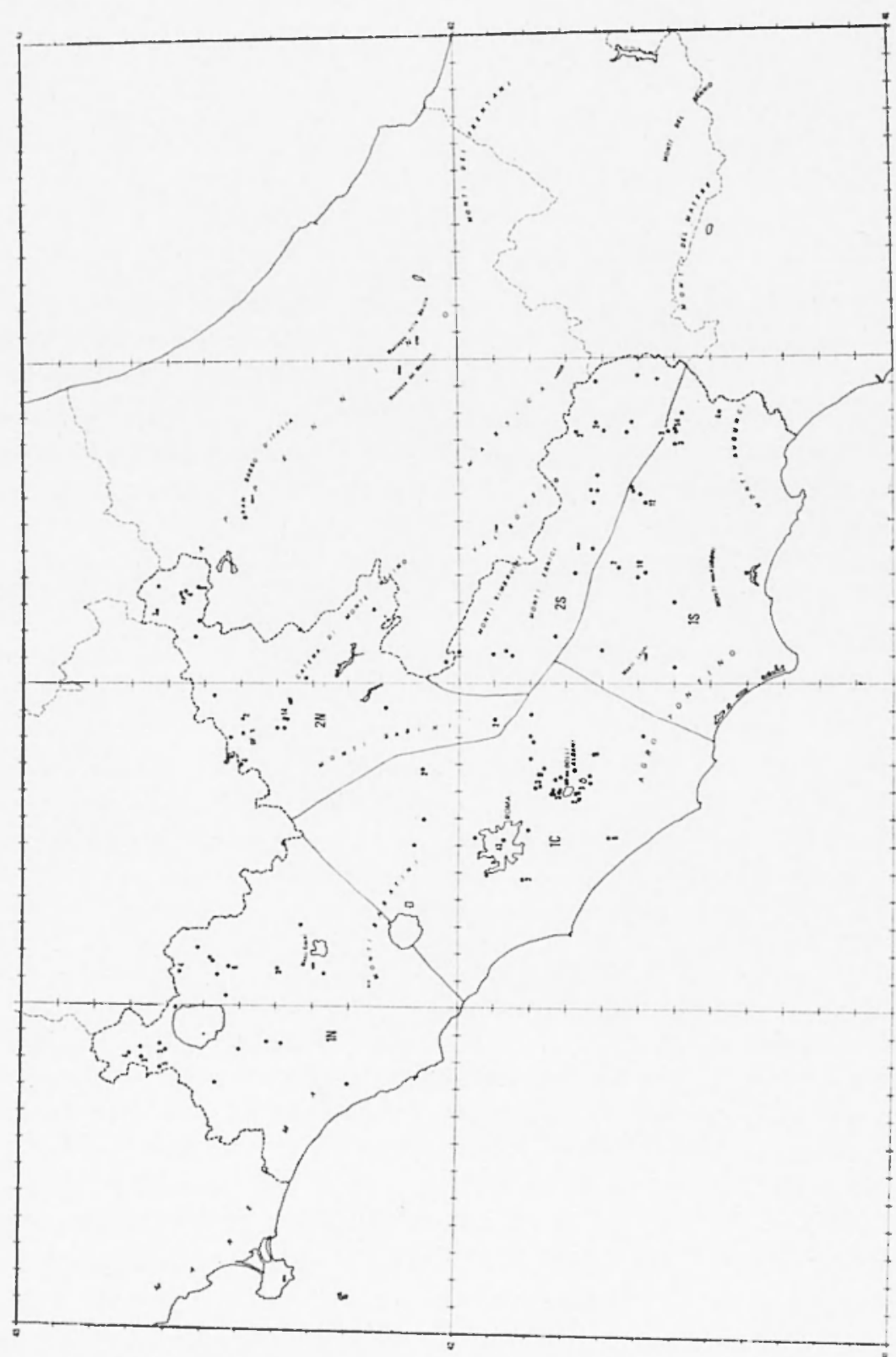

Fig. 1 - Epicentres of earthquakes with $I_{0} \geq$ VI M.C.S. occured in Latium during last 20 centuries. The figure indicates the multiplicities of the events. 
valid for central Italy, in which $I_{0}$ is the intensity at the epicentre. The values of $h$ indicated whith $h$, were derived from the relationship

$$
h=\frac{s_{n}}{\sqrt{10^{\frac{I_{o}-I_{n}}{3}-1}}}
$$

where $s_{n}$ is the radius of the isoseimal of intensity $I_{n}$ using isoseismals found in the literature or constructed to this purpose. When the available information was too poor to construct reliable isoseismals, the $h$ values, indicated with $h_{d}$, were derived considering the distance from the epicentre at which the earthquake had a given intensity. When both the above mentioned methods were possible, the value of the depth given in the table, denoted with $h_{i, d}$, was obtained by averaging the two values of $h_{i}$ and $h_{d}$ weighted with the inverse of the respective errors.

When computable, the half of the maximum spread was taken as the error of the depth; in the other cases the error was calculated by using the mean relative error of the focal depth derived from the same kind of information; isoseismals, distances of both.

The mean relative errors are, for the three cases

$$
\left(\frac{\overline{\Delta h}}{h}\right)_{i}=19 \% \quad ;\left(\frac{\overline{\Delta h}}{h}\right)_{d}=42 \% \quad ;\left(\frac{\overline{\Delta h}}{h}\right)_{i, d}=19 \%
$$

These evaluations do not take into account the errors due to the uncertainties in the definition of $h$.

The magnitude $M$ is expressed in Richter scale and the values derived from microseismic data are indicated with an asterisk; in the absence of these data, when it was possible to reckon the focal depth, the value of the magnitude was calculated by means of equation [1]. 
In cases in which it was impossible to evaluate the focal depth, the magnitude was calculated using Marcelli-Montecchi (1962) equations

$$
\begin{gathered}
M=0.481 I_{\mathrm{o}}+1.407 \quad\left(I_{\mathrm{o}}<\mathrm{VIII}\right) \\
M=0.024 I_{\mathrm{o}}{ }^{2}+0.206 I_{\mathrm{o}}+2.157 \quad\left(I_{\mathrm{o}}>\mathrm{VIII}\right)
\end{gathered}
$$

For relations [3] and [4] the authors have suggested additional corrective terms different for the different geological zones to which they refer; no corrective term had been estimated for Latium because of the poor number of available data.

The 14 earthquakes having an intensity $I_{0} \leq$ VIII occurred in Latium area and whose magnitude was derived on the basis of microseismic data were then used; the parameters relative to the best straight line representing $M$ as a function of $I_{0}$ were calcalculated in order to compare them to those appearing in Marcelli-Montecchi linear relation. The straight line thus obtained has the following equation

$$
M=0.497 I_{\mathrm{o}}+1.058
$$

and is shown in Fig. 2.

The difference between the magnitudes calculated by means of [3] and [5] varies from 0.22 , corresponding to $I_{0}=8$, and 0.25 corresponding to $I_{0}=6$; the considerable spread of the experimental data makes this difference meaningless; in fact the standard deviation of the value $M\left(I_{\mathrm{o}}=0\right)$ amounts to +0.59 .

In order to calculate $M$, relations [3] and [4] where then used without introducing any corrective terms.

The energy $E$ released in the various earthquakes was evaluated using the relation (Di Filippo, Marcelli, 1950).

$$
\log E=9.154+2.147 M
$$




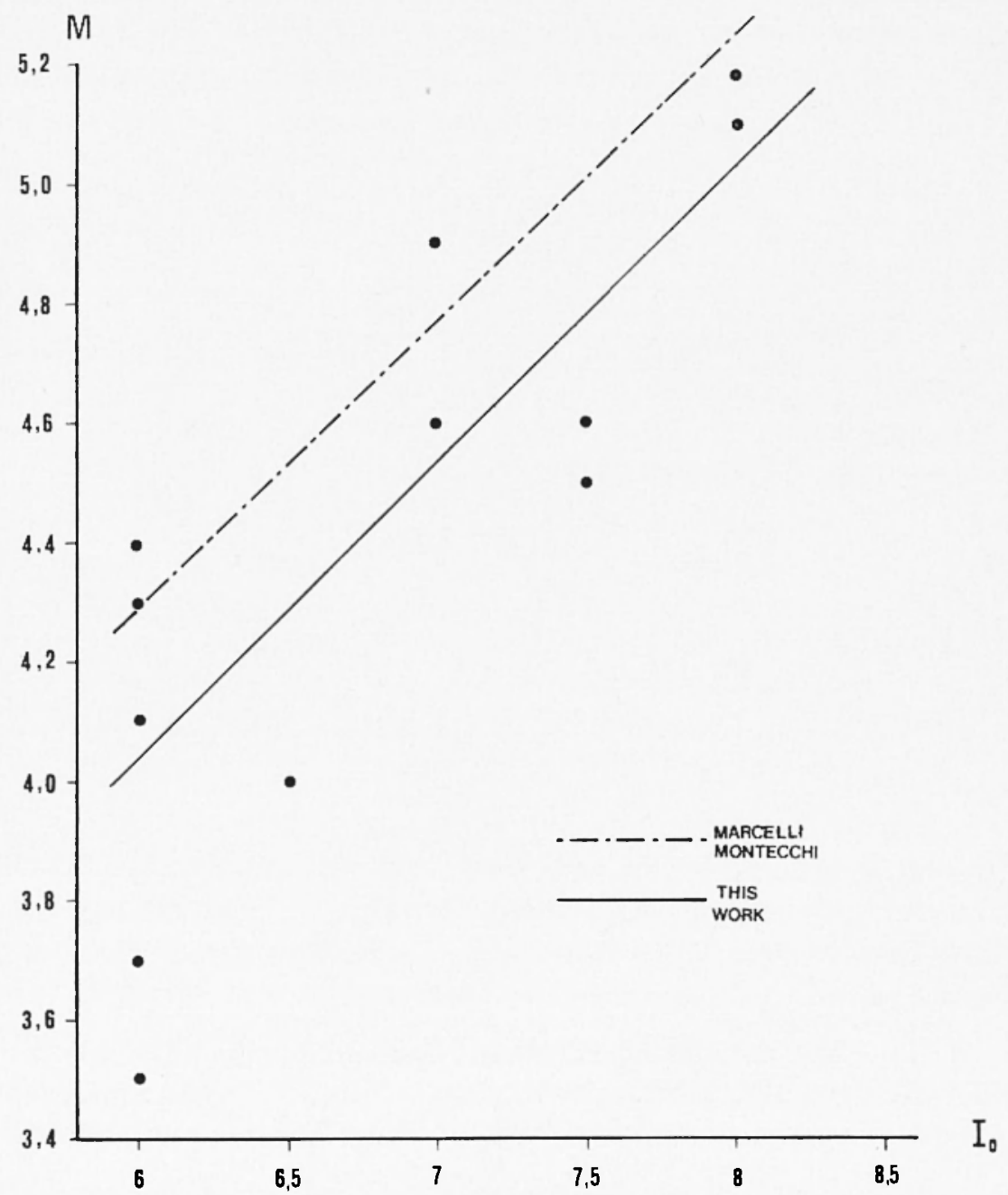

Fig. 2 - Magnitude $M$ as a function of the maximum macroseismic intensity $I_{0}$ according to Marcelli-Montecchi and to this paper. 
In Fig. 1 the epicentres of the earthquakes occurred in Latium in the Christian Era are reported together with the corresponding multiplicities. There are in all 223 events with $I_{0} \geq$ VI and their numerical distribution among the different zones is given in Table 1.

TABLE 1

\begin{tabular}{|c|c|}
\hline ZONE & EARTHQUAKES NUMBER \\
\cline { 2 - 2 } $1 \mathrm{~N}$ & 43 \\
$1 \mathrm{C}$ & 77 \\
$1 \mathrm{~S}$ & 42 \\
$2 \mathrm{~N}$ & 38 \\
$2 \mathrm{~S}$ & 23 \\
\hline
\end{tabular}

According to the catalogue, the magnitude of the considered earthquakes is generally within the range $4.1-5.7$; it is greater than 5.7 only in 8 earthquakes (N. 11, 13, 22, 50, 56, 59, 61, 134) and less than 4.1 in 29 cases corresponding to the $13 \%$ of the total.

3. - The data listed in Table 5 form a useful basis for the identification of typical aspects of seismicity in Latium area with reference to the zones into which this area has been subdivided.

A) For each of the above mentioned five zones the average value of the focal depth was calculated, each value of $h$ being weighted with its error (obviously the meaning of this error is only an indication of the spread of the calculated values of $h$ ). The average values thus obtained are reported in Table 2 .

The value of $\bar{h}$ for the zone $1 \mathrm{~S}$ was calculated ignoring the earthquakes to the south of Monte Cairo in the small triangle 
formed by the localities of Montecassino (Abbey), S. Germano and Villa S. Lucia, the effects of which had a very limited extension. These shocks, indicated with $1 \mathrm{~S}_{\mathrm{L}}$ in the third column of the catalogue, must be considered as originating from a surface focus the depth $\bar{h}$ of which was estimated $1.7 \pm 0.1 \mathrm{Km}$.

TABLE 2

\begin{tabular}{|c|c|}
\hline ZONE & $h \pm \Delta \bar{h}(\mathrm{Km})$ \\
\hline $1 \mathrm{~N}$ & $4.4 \pm 0.6$ \\
$1 \mathrm{C}$ & $5.9+0.3$ \\
$1 \mathrm{~S}$ & $9+2$ \\
$2 \mathrm{~N}$ & $8+2$ \\
$2 \mathrm{~S}$ & $9 \pm 2$ \\
\hline
\end{tabular}

As it turns out from Table 3, the average focal depth, proceeding from North to South along the volcanic belt, increases. Furthermore, one can see that zones $1 \mathrm{~N}$ and $1 \mathrm{C}$ are characterized by values of $h$ less than those of the remaining Latium zones; one has in fact

$$
\begin{aligned}
& \bar{h}_{1 \mathrm{~N}+\mathrm{IC}}=5.4 \pm 0.5 \mathrm{Km} \\
& \bar{h}_{\mathrm{is}+2 \mathrm{~N}+2 \mathrm{~s}}=9 \pm 2 \mathrm{Km}
\end{aligned}
$$

The last value is in agreement with the result obtained by Kárnik 1969, indicating, for central Italy, a characteristic focal depth of $10-15 \mathrm{Km}$, showing for the earthquakes in zones $1 \mathrm{~S}, 2 \mathrm{~N}$ and $2 \mathrm{~S}$ aspects which are typically connected to tectonic-orogenetic phenomena in the Apennines. 
B) As it is known, the $b$ value appearing in the relation (Gutenberg, Richter, 1954)

$$
\log N=a-b M
$$

in which $N$ is the number of the earthquakes having a magnitude falling in a given range around $M$, is a quantity expressing the seismic activity of a region.

The $b$ value was evaluated by Karník, 1969, as 1.03 for central Italy. It would be interesting to specify this value for the different zones considered in Latium area.

In Table 3 the values of the parameters $a$ and $b$ appearing in relation [7] as calculated by the least squares method are reported $\left({ }^{*}\right)$.

TABLE 3

\begin{tabular}{|c|c|c|}
\hline ZONE & $a \pm \sigma_{a}$ & $b \pm \sigma_{b}$ \\
\hline $1 \mathrm{~N}$ & $4.6 \pm 0.1$ & $0.74+0.01$ \\
$1 \mathrm{C}$ & $4.8 \pm 0.2$ & $0.74 \pm 0.04$ \\
$1 \mathrm{~S}+1 \mathrm{~S}_{\mathrm{L}}$ & 3.1 & 0.40 \\
\hline $2 \mathrm{~N}+2 \mathrm{~S}$ & $4.5 \pm 0.2$ & $0.67 \pm 0.03$ \\
$1 \mathrm{~N} \pm 1 \mathrm{C}$ & $6.1 \pm 0.2$ & $0.98 \pm 0.04$ \\
\hline
\end{tabular}

Earthquakes occurred since A.D. 1200 were taken into account, the previous information being hardly reliable. When only two experimental points were available it was impossible to calculate the standard deviations $\sigma_{a}$ and $\sigma_{b}$ : however, it seems

(*) Relation [7] is valid in the range of the $M$ values which are more frequent in the considered region. 
reasonable to consider them of the same order of the calculated ones. Obviously, also the earthquakes relative to the surface focus of Montecassino are to be included in the group of those occurred in region $1 \mathrm{~S}$.

As is possible to see from Table 3 the $b$ value decreases abruptly, along the Latium volcanic belt, in the zone 1S.

The available data were too scarce to detect significantly a similar effect along the western belt of the region; as an indication the $b$ value for the zones $2 \mathrm{~N}$ and $2 \mathrm{~S}$ considered as a whole was then calculated.

It is also possible to see from Table 3 that zones $1 \mathrm{~N}$ and $1 \mathrm{C}$ are characterized by $b$ values greater than those relative to the remaining zones.

Both the observed effects are in agreement with those concerning the focal depths; in fact deeper events release more energy thus lowering the absolute value of $b$ in relation [7]. The $b$ value relative to the whole Latium area turns out to be $b_{L}=0.90 \pm 0.03$.

The value which refers to the very volcanic belt (zone $1 \mathrm{~N}+$ 1C) is in the best agreement with the one given by Kárnik.

C) A further elaboration of the collected data was carried out in order to identify the time behaviour of the seismicity. The migration of the average of the epicentres for the earthquakes occurred in each zone in given periods was taken as an indication of this evolution (Biagi et al., 1974).

The values of latitude $\varphi$ and longitude $\lambda$ of the average epicentres (Table 4) were calculated effecting a weighted average the magnitude being the weight. The periods were fixed in such a way as to include comparable amounts of shocks.

The migration of the activity in every single zone is plotted in Fig. 3 starting from A.D. 1200; the period before A.D. 1200 was not taken into consideration because of the poor reliability of the available information concerning the dislocation of the epicentres.

From the figure one can see at first that in each zone it is possible to identify a direction (two for zone $2 \mathrm{~N}$ ) along which 
M. ALESSIO - P. F. BIAGI

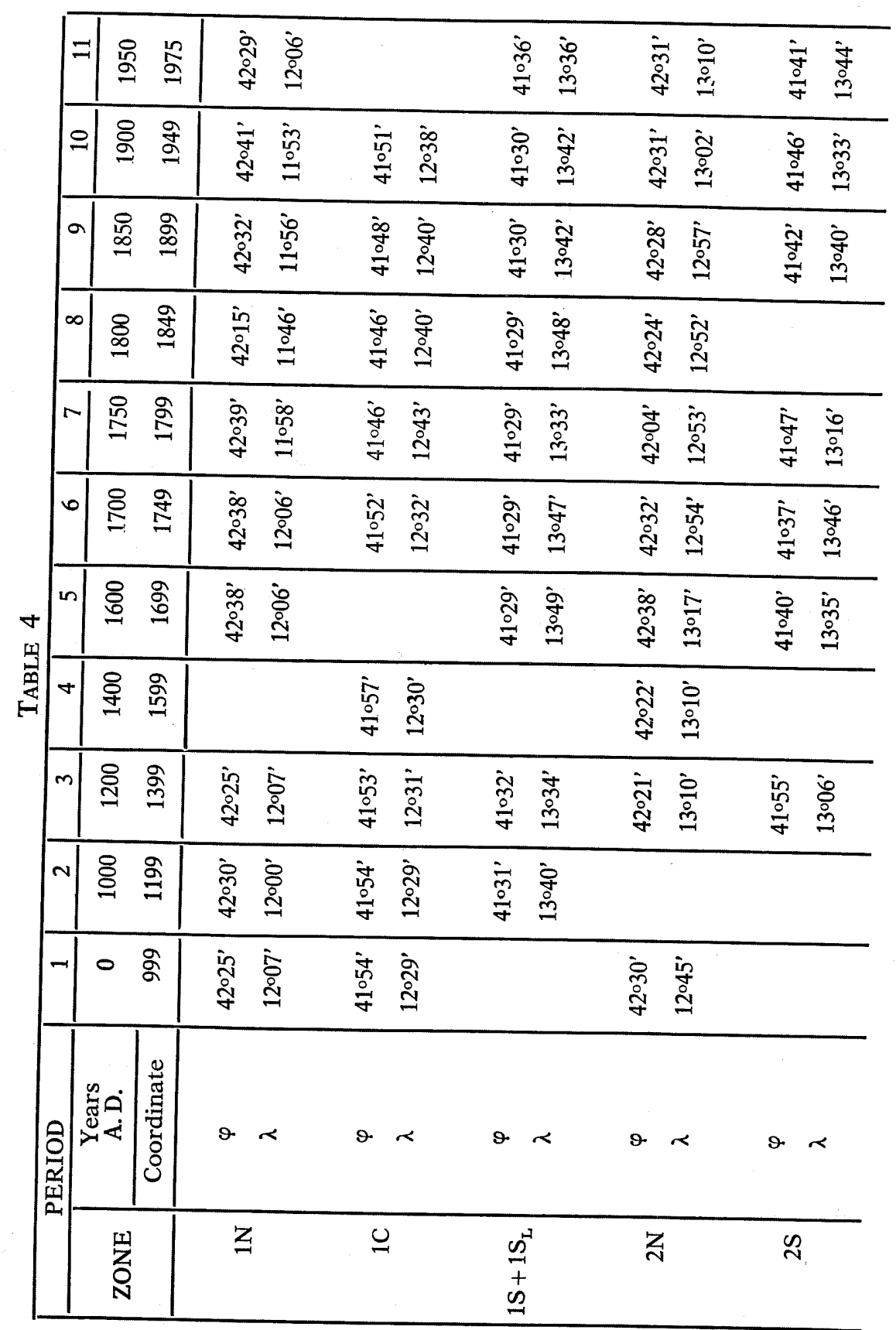




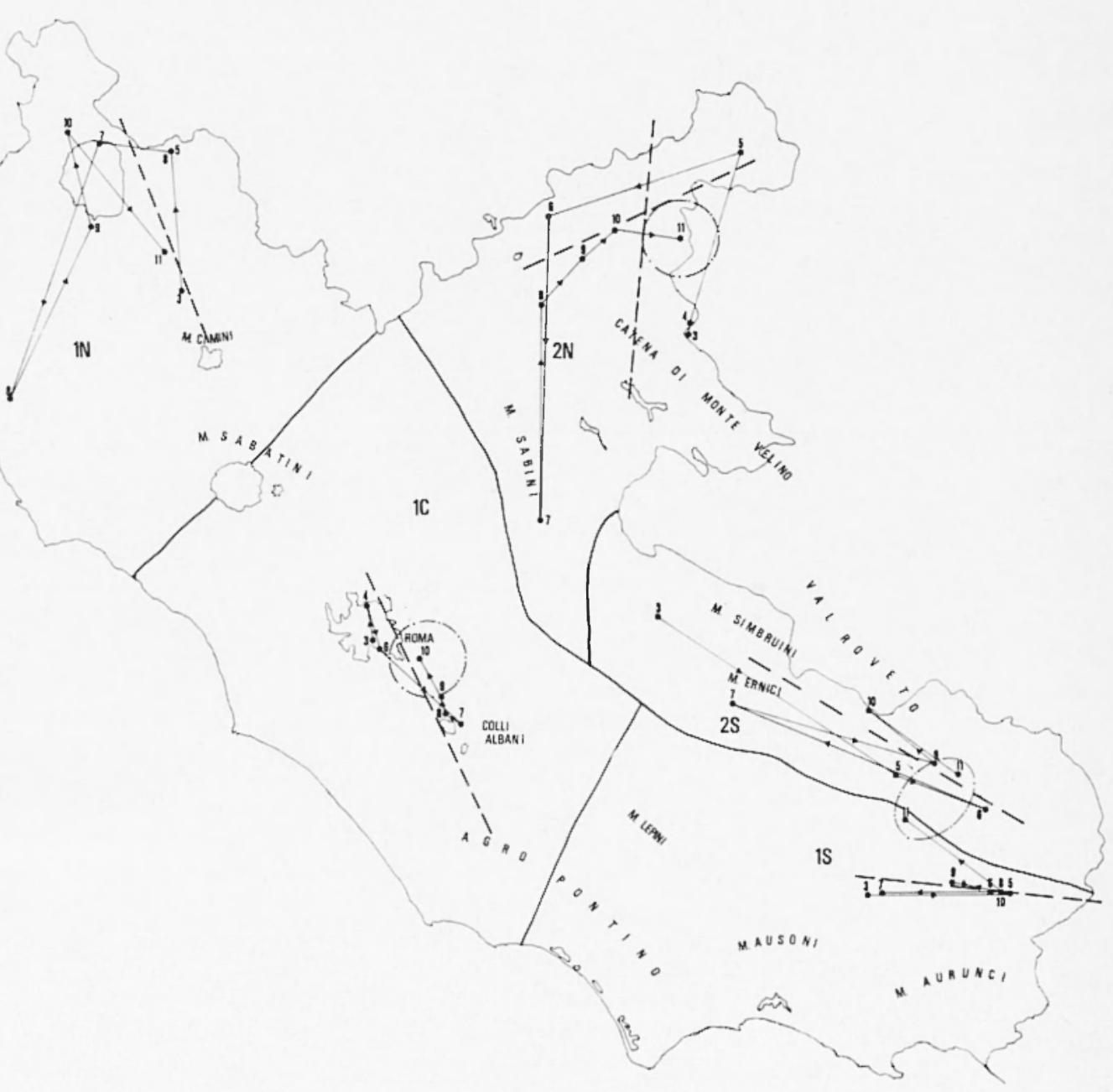

Fig. 3 - Activity migration in Latium region since A.D. 1200. Dotted lines represent preferential directions of migration. The areas delimited by point-dot lines are those of more recent convergence of the seismic activity. 


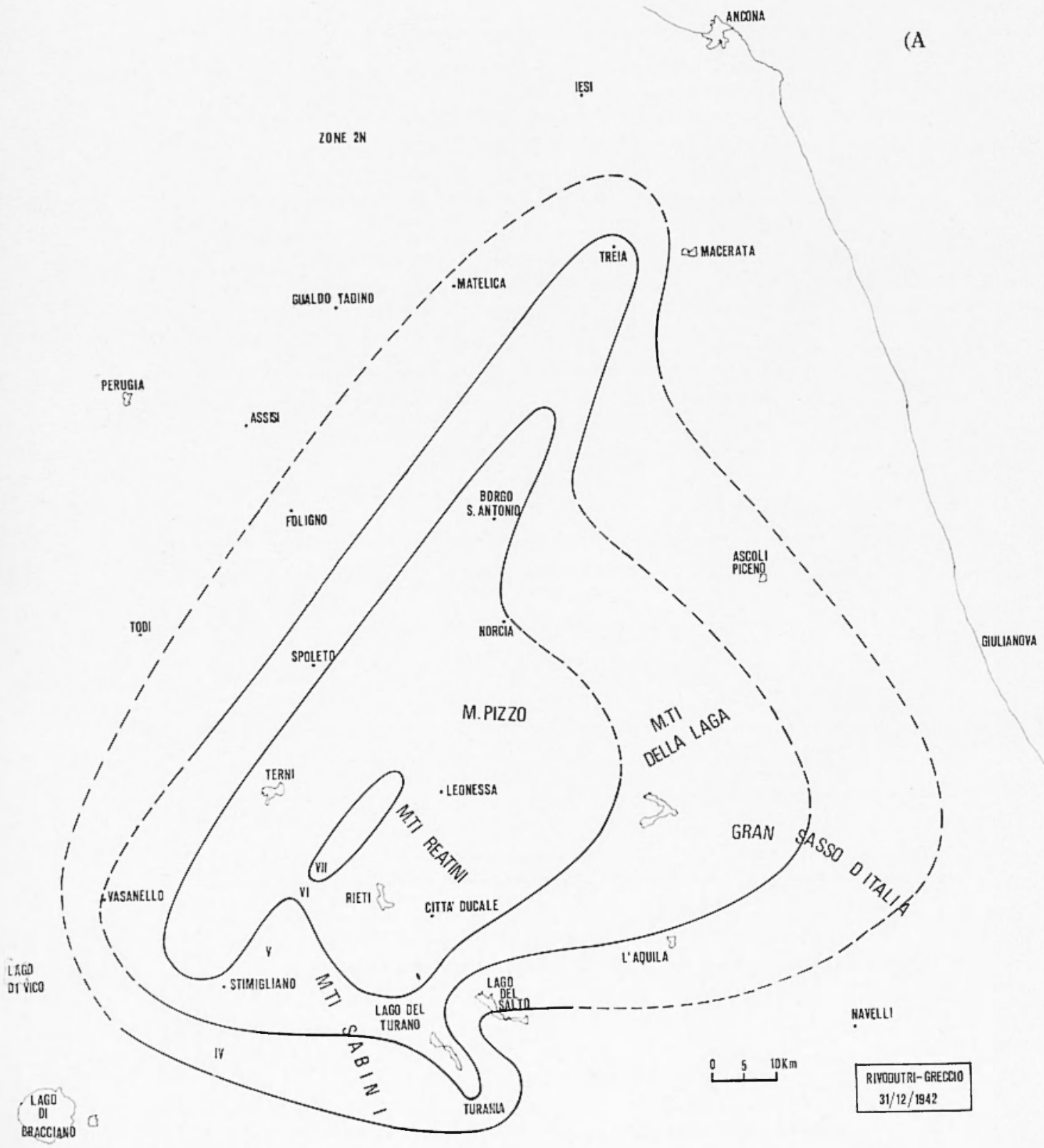

I11:-1

Fig. 4 A, B, C - Isoseismals of seme earthquakes occurred in Latium. 
201:E li:

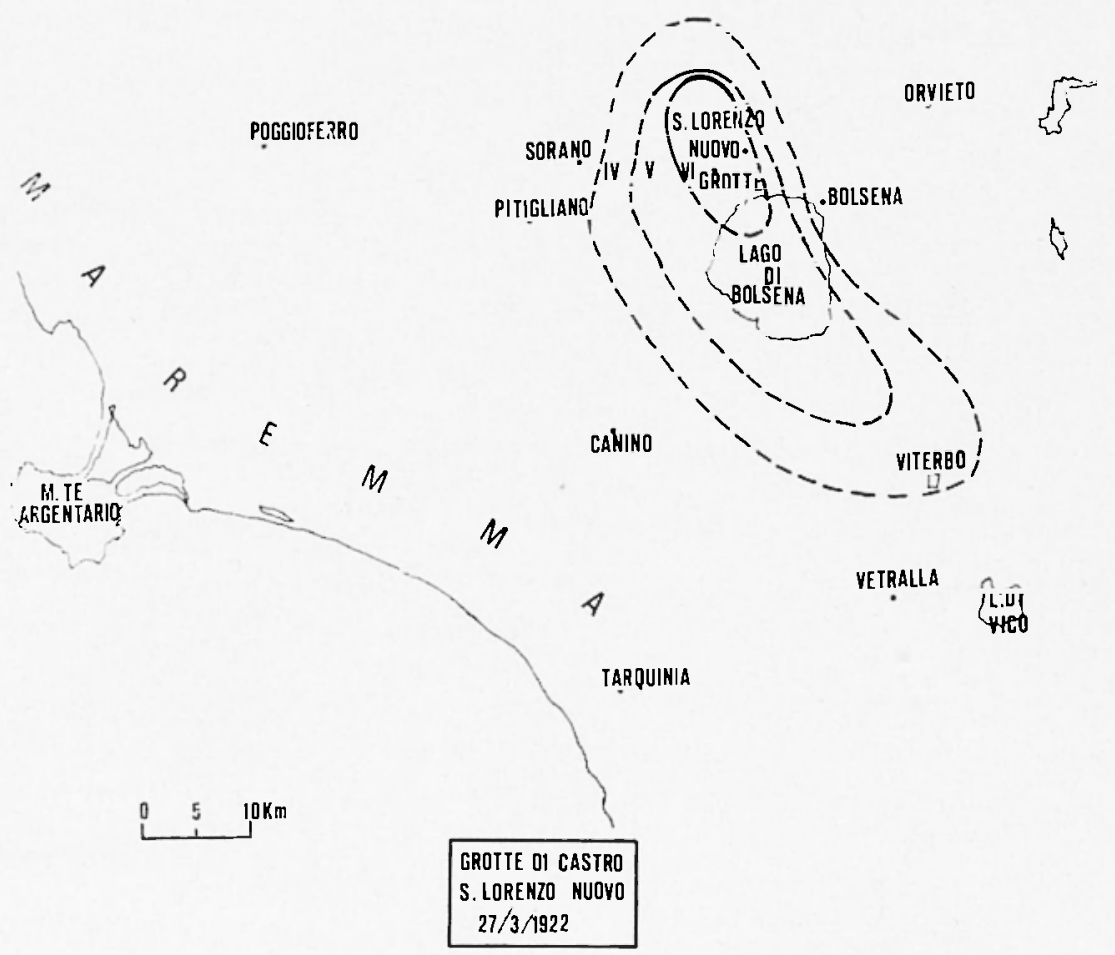

preferentially the average epicentre migrates (dotted lines in Fig. 3).

The parameters relative to these lines were derived as a weighted average of those of the segments joining the average epicentre of each period with the next one; the assumed weight was the sum of the numbers of earthquakes used to calculate the average epicentres forming the end points of each segment.

A comparison in each zone of these directions with the elliptical elongation of the isoseismals (Fig. 4A, B, C) shows a substantial agreement, thus showing that the anisotropy in the elastic characteristics of the crystal rocks seems to favour coherently the migration of the epicenter. 


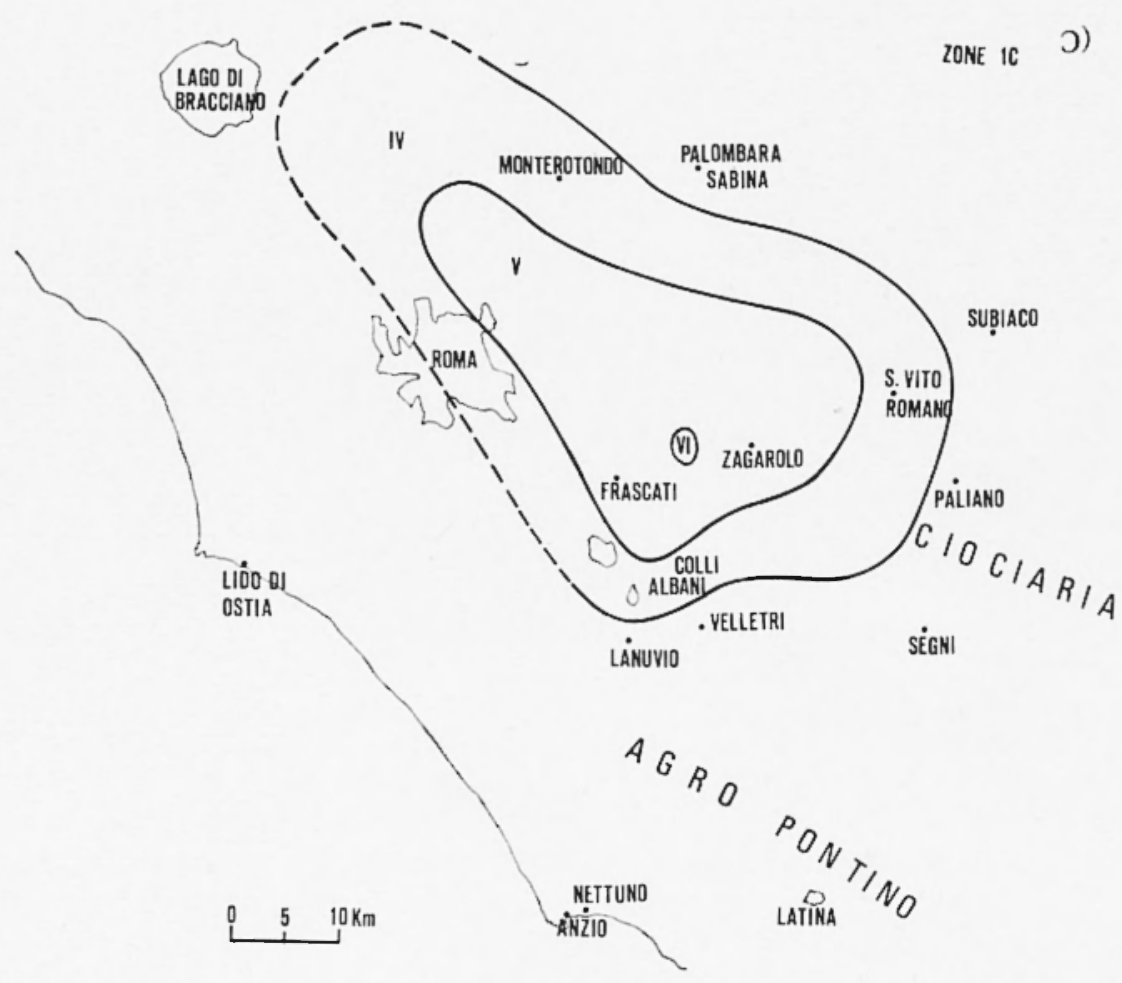

It also results form Fig. 3 that in the more recent periods there has been in zone $2 \mathrm{~N}$ a tendency of activity to shift along a preferential line in a given direction; a similar, though less important behaviour, is also present in zone $1 \mathrm{C}$. In the adjacent zones $1 \mathrm{~S}$ and $2 \mathrm{~S}$ the average epicentre seems to have a tendency to migrate towards a same area.

These effects allow the approximate identification of zones, only indicatively delimited in Fig. 3 , in which it might be convenient to undertake an experimental investigation, for instance on the variation of the ratio of compressional-to-shear wave ve- 


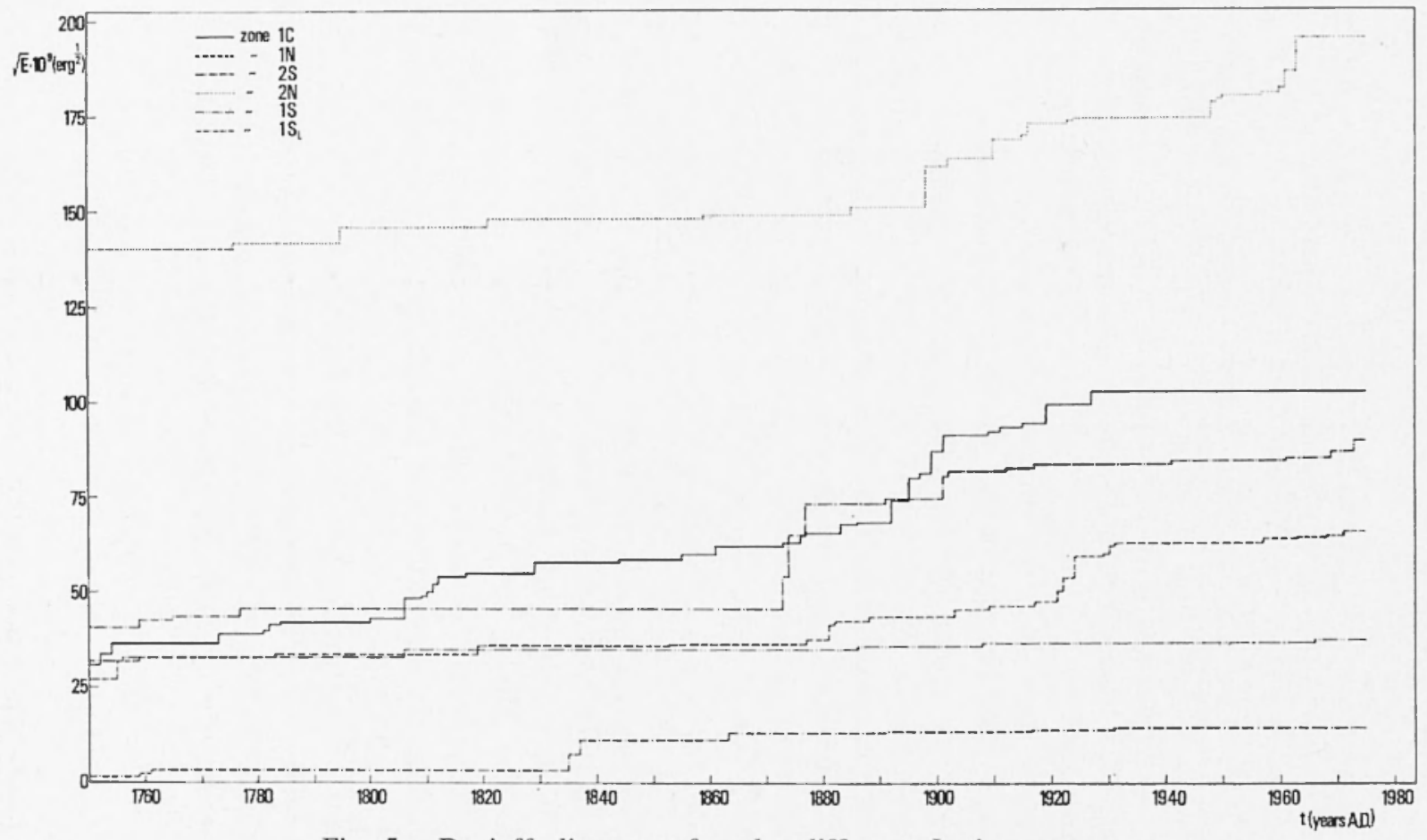

Fig. 5 - Benioff diagrams for the different Latium zones since A.D. 1750. The cumulative values of $\sqrt{E}$ until A.D. 1750 can be read on the vertical axis on the left. 
locities on the variation of radon concentration in wall water, etc.

D) In Fig. 5 Benioff, 1951, plots of elastic strains since 1750 for the considered zones are reported.

As is seen, the elastic-strain energy has revealed itself in zone 1S fairly regularly in time, without any violent collapses; the same can be observed for the area $1 S_{L}$ with the exception of the period 1835-1863. In the remaining zones, throughout the period from 1865 to 1935 approximately, the accumulated strain energy was released in a convulsive way, showing an instability in the elastic field in almost the whole Latium region.

In the years before the period above there were no violent and sudden releases of energy in zones $2 \mathrm{~N}, 2 \mathrm{~S}, 1 \mathrm{~N}$ whereas a convulsive and irregular instability in the elastic field was already active in zone 1C; after the year 1935 the situation in the above mentioned zones seems to invert, an accumulation of energy appearing in zone $1 \mathrm{C}$, which is still present today.

4. - In conclusion, the study carried out up to now has allowed the differentation of some phenomenologic aspects of the seismicity in different zones of Latium region. In particular the focal depth turned out to be less in the volcanic belt (from Bolsena to the Alban Hills) than in the remaining zones, also increasing from North to South; the $b$ value showed an opposite behaviour. The time evolution of the seismic activity showed in some cases well defined characteristics, thus indicating approximately areas of particular interest for future experimental investigations. With a better geotectonic knowledge of Latium it will be possible to have a complete picture of the correlations between elastic-strain energy and structural characteristics.

\section{ACKNOWLEDGEMENTS}

This work has been carried out as a part of Geolazio-program supported by University of Rome. 


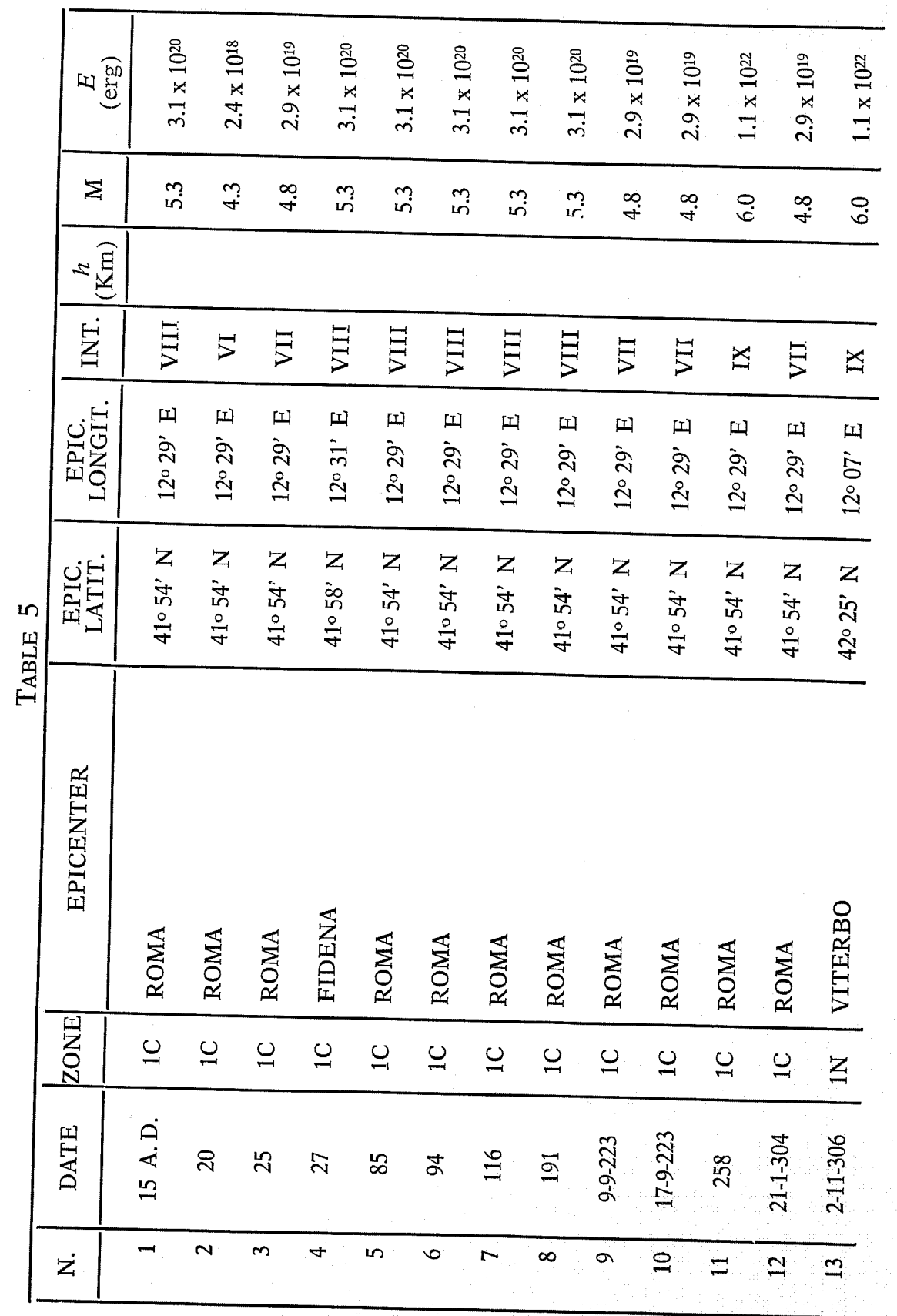




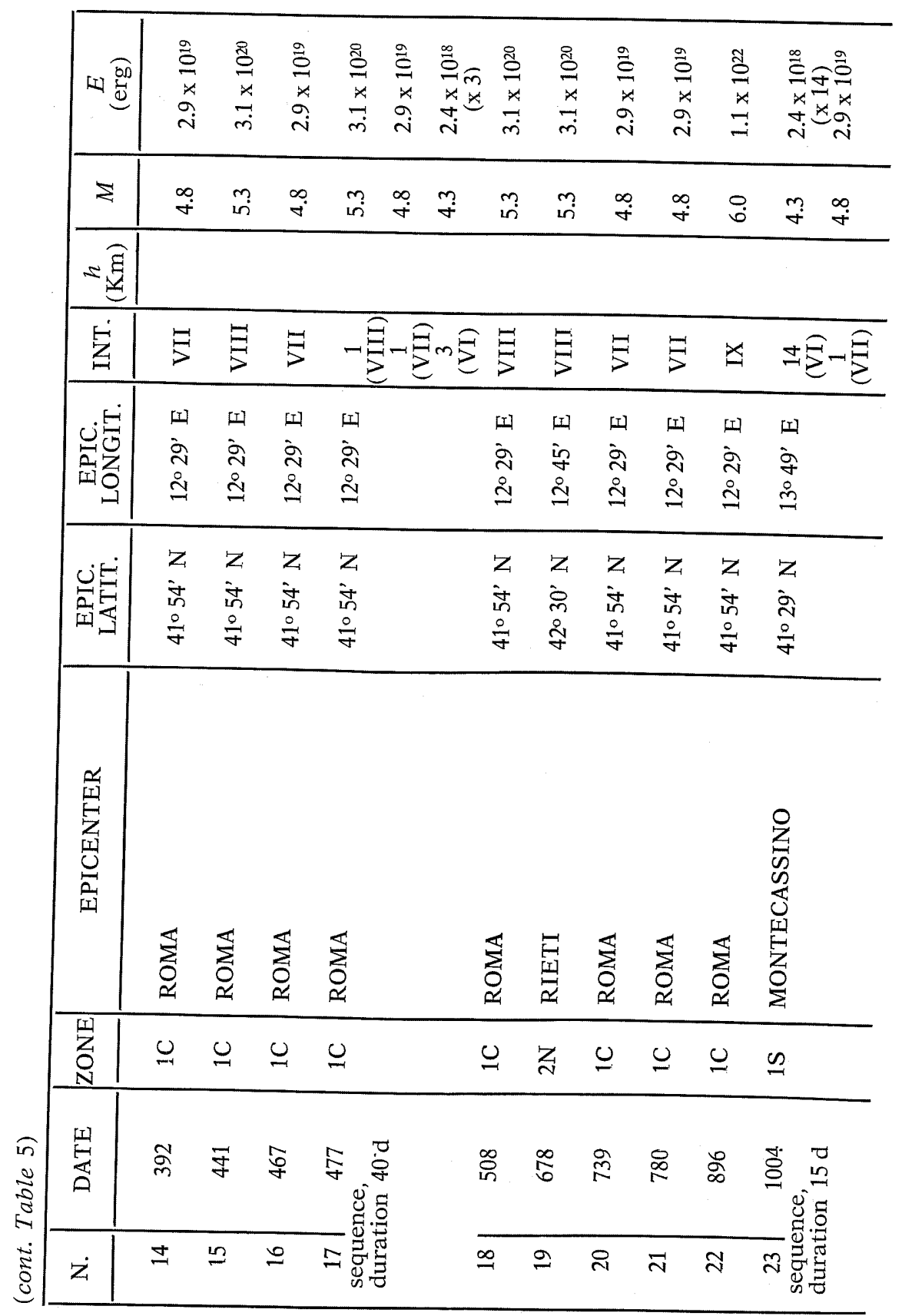




\begin{tabular}{|c|c|c|c|c|c|c|c|c|}
\hline 过惢 & 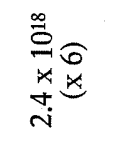 & 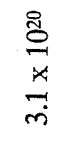 & 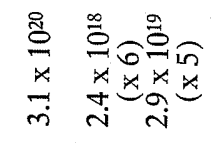 & 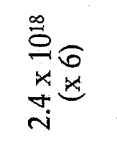 & $\begin{array}{l}\stackrel{a}{0} \\
\stackrel{x}{j}\end{array}$ & 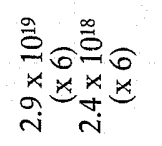 & $\begin{array}{l}a \\
\stackrel{a}{0} \\
x \\
a \\
\end{array}$ & $\begin{array}{l}\stackrel{a}{\sigma} \\
\stackrel{x}{a} \\
a\end{array}$ \\
\hline$\Sigma$ & $\stackrel{m}{*}$ & in & $m \stackrel{m}{\sharp} \stackrel{\infty}{+}$ & $\stackrel{m}{\forall}$ & $\stackrel{\infty}{+}$ & $\stackrel{\infty}{+} \stackrel{n}{+}$ & $\stackrel{\infty}{+}$ & $\stackrel{\infty}{+}$ \\
\hline 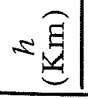 & & & & & & & & \\
\hline 点 & $\widehat{0}$ & $\Xi$ & 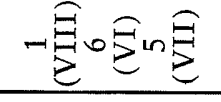 & $\stackrel{8}{Z}$ & 5 & 㽗是 & 5 & 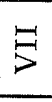 \\
\hline 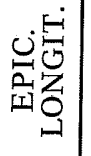 & $\begin{array}{l}\text { 디 } \\
\grave{ } \\
\stackrel{\Xi}{\beth}\end{array}$ & $\begin{array}{l}\text { 되 } \\
\dot{8} \\
\stackrel{0}{0}\end{array}$ & $\begin{array}{l}\text { 디 } \\
\dot{\infty} \\
\stackrel{m}{2} \\
\stackrel{m}{=}\end{array}$ & $\begin{array}{l}\text { ㄸ } \\
\text { के } \\
\stackrel{+}{\sim}\end{array}$ & 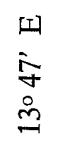 & $\begin{array}{l}\text { 미 } \\
\dot{a} \\
\dot{+} \\
\stackrel{2}{2}\end{array}$ & $\begin{array}{l}\text { 뙤 } \\
\ddot{\sim} \\
\stackrel{0}{2}\end{array}$ & $\begin{array}{l}\text { 디 } \\
\stackrel{-}{N} \\
\stackrel{2}{2}\end{array}$ \\
\hline 记䛼㫕 & $\begin{array}{l}Z \\
\dot{Z} \\
\dot{7} \\
\stackrel{7}{7}\end{array}$ & $\begin{array}{l}Z \\
\dot{z} \\
\dot{m} \\
\stackrel{+}{y}\end{array}$ & $\begin{array}{l}z \\
\dot{y} \\
m \\
0 \\
\dot{q}\end{array}$ & $\begin{array}{l}\text { Z } \\
\text { ેㅣ } \\
\stackrel{0}{\forall}\end{array}$ & $\begin{array}{l}z \\
\dot{2} \\
\stackrel{2}{7}\end{array}$ & $\begin{array}{l}z \\
\dot{ก} \\
\vdots \\
\dot{y}\end{array}$ & $\begin{array}{l}Z \\
\dot{\infty} \\
m \\
\stackrel{0}{\forall}\end{array}$ & $\begin{array}{l}\text { Z } \\
\dot{\infty} \\
\text { ○ } \\
\stackrel{+}{\forall}\end{array}$ \\
\hline 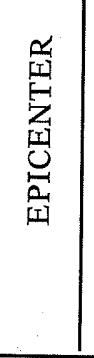 & $\sum_{\substack{1 \\
\hdashline}}^{\mathbb{1}}$ & 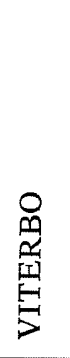 & 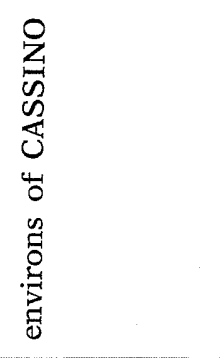 & 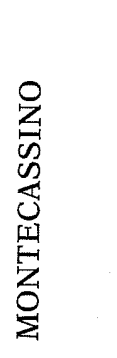 & 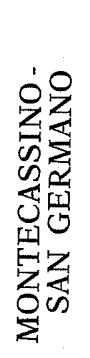 & 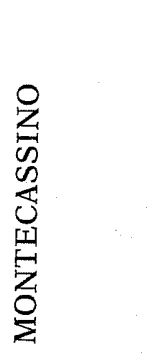 & 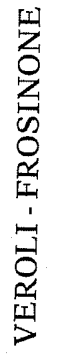 & 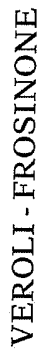 \\
\hline $\begin{array}{l}\text { II } \\
\text { 告 } \\
\text { N }\end{array}$ & $\stackrel{\cup}{\cup}$ & 吕 & 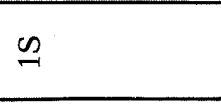 & $\approx$ & $\mathrm{S}^{2}$ & 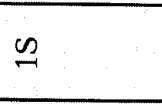 & 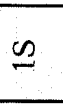 & $\stackrel{\sim}{\leadsto}$ \\
\hline 豆 & 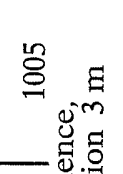 & $\stackrel{\Xi}{\Xi}$ & $\stackrel{\text { 굴 }}{=}$ & 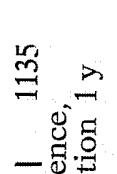 & $\stackrel{9}{=}$ & 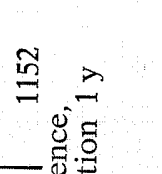 & $\frac{8}{8}$ & $\frac{5}{\frac{5}{7}}$ \\
\hline$Z$ & 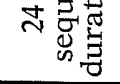 & $\stackrel{\llcorner}{\sim}$ & 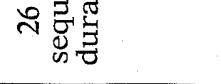 & ล & $\stackrel{\sim}{\sim}$ & 구류 & $\stackrel{p}{ }$ & $\bar{m}$ \\
\hline
\end{tabular}


M. ALESSIO - P. F. BIAGI

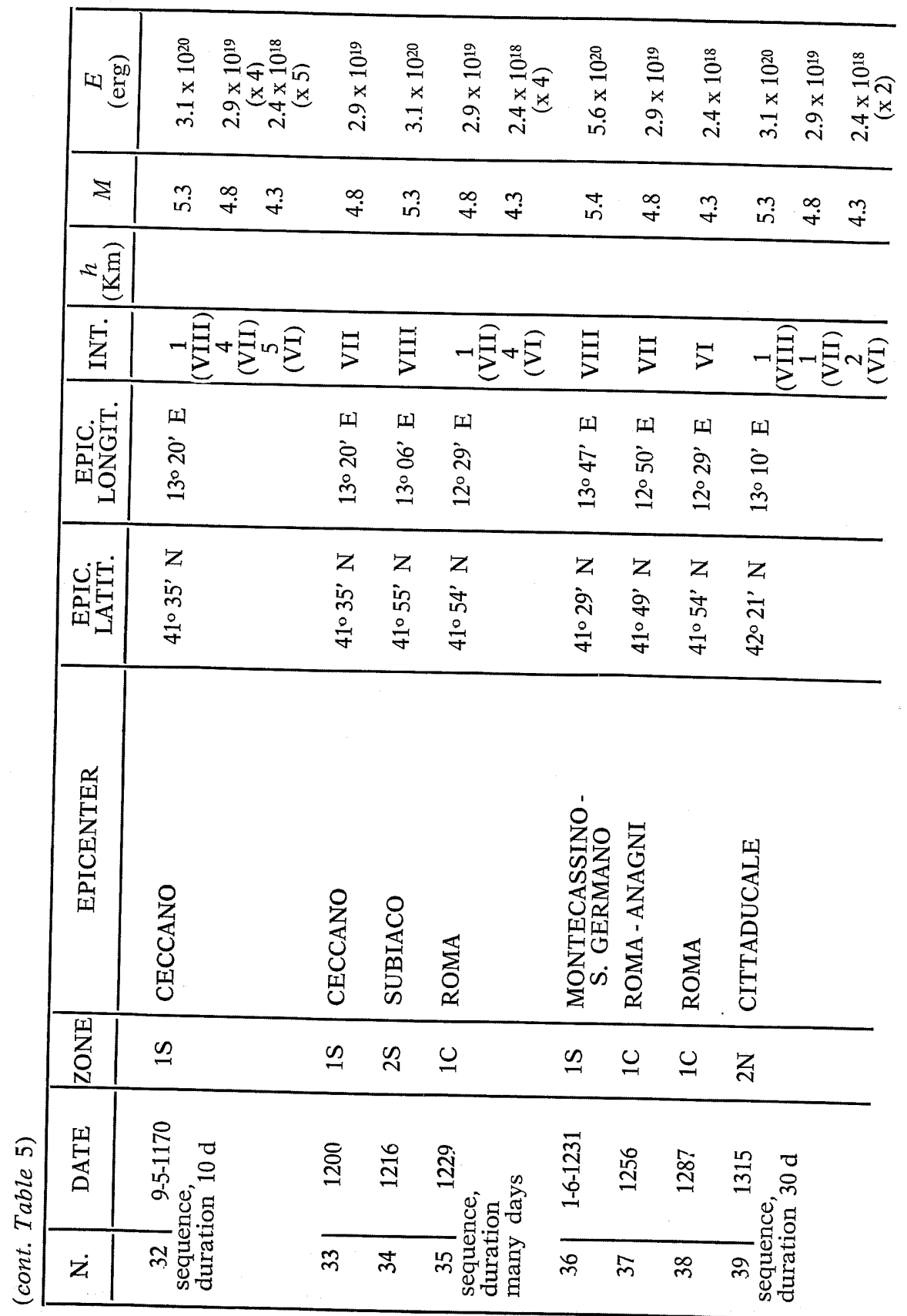




\begin{tabular}{|c|c|c|c|c|c|c|c|c|c|c|}
\hline 以寅 & $\frac{\stackrel{a}{o}}{\check{a}}$ & 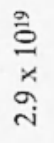 & $\frac{\stackrel{d}{0}}{\frac{\check{x}}{m}}$ & 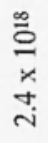 & $\begin{array}{l}\stackrel{a}{\sigma} \\
\ddot{\alpha} \\
\ddot{\alpha}\end{array}$ & $\begin{array}{l}\stackrel{\infty}{\varrho} \\
\ddot{x} \\
\stackrel{d}{u}\end{array}$ & $\begin{array}{l}\stackrel{a}{0} \\
\ddot{x} \\
a \\
\text { vi }\end{array}$ & 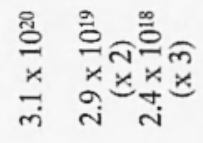 & 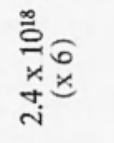 & $\begin{array}{l}\stackrel{\infty}{\sigma} \\
\stackrel{0}{*} \\
\dot{+} \\
\text { i }\end{array}$ \\
\hline$\Sigma$ & $\stackrel{\infty}{+}$ & $\stackrel{\infty}{+}$ & in & $\stackrel{m}{+}$ & $\stackrel{\infty}{+}$ & $\stackrel{m}{+}$ & $\stackrel{\infty}{+}$ & $m \stackrel{m}{i} \stackrel{m}{+}$ & & $\stackrel{m}{+}$ \\
\hline$=\widehat{\text { 国 }}$ & & & & & & & 욱 & & & \\
\hline 它 & 5 & 5 & $\Xi$ & 5 & 马 & 5 & $\Xi$ & 一寻的刍 & & 5 \\
\hline 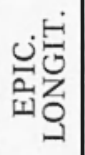 & $\begin{array}{l}\text { 피 } \\
\vdots \\
\vdots \\
\vdots\end{array}$ & 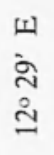 & $\begin{array}{l}\text { પ1 } \\
\text { ปे } \\
\text { ปे }\end{array}$ & $\begin{array}{l}\text { ㄴ } \\
\text { ஸे } \\
\text { さे }\end{array}$ & $\begin{array}{l}\text { w } \\
\text { ते } \\
\text { ปे }\end{array}$ & $\begin{array}{l}\text { 凹1 } \\
\text { సे } \\
\text { ปे }\end{array}$ & 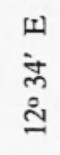 & $\begin{array}{l}\text { 띠 } \\
\text { 음 } \\
\stackrel{2}{2}\end{array}$ & $\begin{array}{l}11 \\
\text { 음 } \\
\text { 임 }\end{array}$ & $\begin{array}{l}\text { w } \\
i \\
\dot{2}\end{array}$ \\
\hline 峁旨 & $\begin{array}{l}z \\
\text { in } \\
\stackrel{+}{y}\end{array}$ & $\begin{array}{l}z \\
\dot{z} \\
\text { in } \\
0 \\
\dot{y}\end{array}$ & $\begin{array}{l}\text { Z } \\
\text { ìे } \\
\stackrel{\circ}{y}\end{array}$ & $\begin{array}{l}\text { Z } \\
\dot{y} \\
\stackrel{0}{0} \\
\stackrel{y}{+}\end{array}$ & $\begin{array}{l}\text { Z } \\
\dot{i} \\
\text { in } \\
\frac{0}{y}\end{array}$ & $\begin{array}{l}z \\
\dot{H} \\
\stackrel{5}{7}\end{array}$ & $\begin{array}{l}Z \\
\text { in } \\
\text { o } \\
\text { ‡े }\end{array}$ & $\begin{array}{l}Z \\
\text { Ż } \\
\stackrel{\text { Iे }}{y}\end{array}$ & $\begin{array}{l}\text { Z } \\
\text { స̃ } \\
\text { సे }\end{array}$ & $\begin{array}{l}z \\
\infty \\
\infty \\
\stackrel{y}{y}\end{array}$ \\
\hline 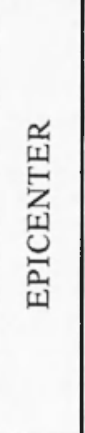 & 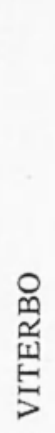 & 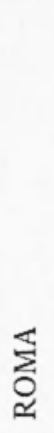 & 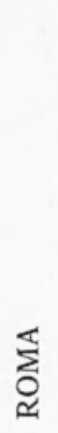 & 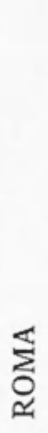 & 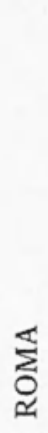 & 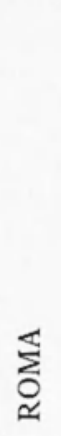 & 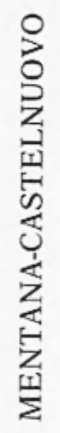 & 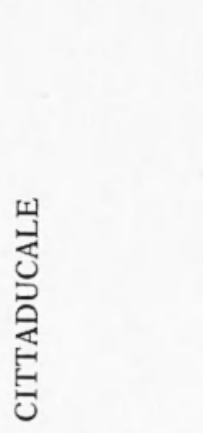 & 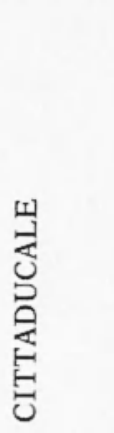 & 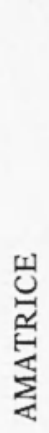 \\
\hline $\begin{array}{l}\text { 믹 } \\
\text { 읏 } \\
\end{array}$ & $\underline{Z}$ & 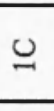 & 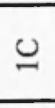 & $\underline{\cup}$ & 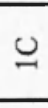 & $\underline{U}$ & $\underline{U}$ & Z & Z & Z্র \\
\hline$\stackrel{\text { 点 }}{\stackrel{\Delta}{日}}$ & ్ి & $\begin{array}{l}\vec{\sim} \\
\stackrel{\sim}{2}\end{array}$ & 点 & 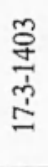 & $\stackrel{\stackrel{2}{ \pm}}{ \pm}$ & 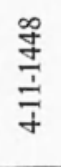 & $\frac{\text { 号 }}{\frac{1}{2}}$ & 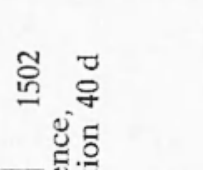 & 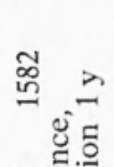 & $\frac{\text { ஸे }}{\frac{d}{\infty}}$ \\
\hline$z \dot{z}$ & 우 & ₹ & $\mathcal{Y}$ & $\dddot{F}$ & J & $\stackrel{\text { ก) }}{+}$ & 웅 & 午忌藍 & 趈苞 & g \\
\hline
\end{tabular}


M. ALESSIO - P. F. BIAGI

\begin{tabular}{|c|c|c|c|c|c|c|c|c|c|c|c|}
\hline 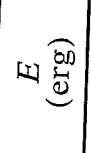 & 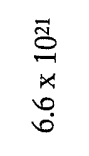 & 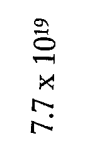 & 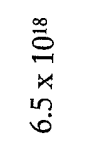 & $\begin{array}{l}\stackrel{\infty}{0} \\
\stackrel{0}{u} \\
\dot{x} \\
\stackrel{+}{i}\end{array}$ & $\begin{array}{l}\stackrel{\infty}{\partial} \\
\stackrel{x}{x} \\
\stackrel{+}{i}\end{array}$ & $\begin{array}{l}\stackrel{a}{0} \\
\dot{x} \\
\stackrel{a}{v}\end{array}$ & 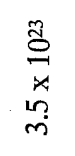 & $\begin{array}{l}\stackrel{9}{0} \\
\not{x} \\
\stackrel{a}{i}\end{array}$ & $\begin{array}{l}\stackrel{\infty}{0} \\
\stackrel{1}{x} \\
\stackrel{x}{+} \\
\sim\end{array}$ & 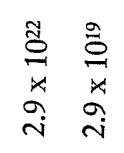 & 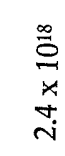 \\
\hline$\Sigma$ & के. & in & $\stackrel{\operatorname{n}}{+}$ & $\stackrel{m}{*}$ & $\ddot{q}$ & $\stackrel{\infty}{+}$ & $\tilde{0}$ & $\stackrel{\infty}{\forall}$ & $\ddot{q}$ & $\underset{\vartheta}{\stackrel{\infty}{*}} \stackrel{\infty}{+}$ & $\stackrel{m}{*}$ \\
\hline$\approx \widehat{\Xi}$ & $\approx$ & & & & & & $\infty \theta$ & & & $\stackrel{0}{ت}$ & \\
\hline 葛 & $\stackrel{\leftrightarrow}{コ}$ & 岕ま & $\stackrel{\text { 今゙ }}{>}$ & $\stackrel{1}{>}$ & 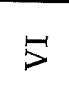 & 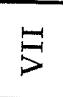 & $x$ & 5 & 5 & $-\sqrt[x]{g}-5$ & 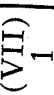 \\
\hline 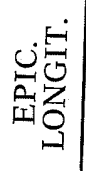 & $\begin{array}{l}{[1]} \\
i \\
\stackrel{1}{1} \\
\stackrel{0}{=}\end{array}$ & $\begin{array}{l}\text { II } \\
\stackrel{i}{=} \\
\stackrel{i}{=}\end{array}$ & $\begin{array}{l}\text { II } \\
\stackrel{1}{2} \\
\stackrel{0}{2}\end{array}$ & $\begin{array}{l}\text { 띠 } \\
\dot{\alpha} \\
\dot{0} \\
\dot{2}\end{array}$ & $\begin{array}{l}\text { 피 } \\
\dot{a} \\
\dot{+} \\
\stackrel{9}{-}\end{array}$ & $\begin{array}{l}\text { 디 } \\
\dot{a} \\
\stackrel{+}{2}\end{array}$ & $\begin{array}{l}\text { 디 } \\
\text { in } \\
\text { ஜ } \\
\stackrel{m}{=}\end{array}$ & $\begin{array}{l}\text { 되 } \\
\text { के } \\
\stackrel{+}{0}\end{array}$ & $\begin{array}{l}\text { 피 } \\
\dot{8} \\
\stackrel{8}{0} \\
\stackrel{0}{0}\end{array}$ & $\begin{array}{l}\text { 피 } \\
\text { 옹 } \\
\stackrel{2}{\Xi}\end{array}$ & \\
\hline 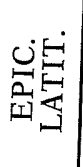 & $\begin{array}{l}z \\
\infty \\
\infty \\
\stackrel{y}{y}\end{array}$ & $\begin{array}{l}Z \\
\dot{\infty} \\
\dot{m} \\
\stackrel{y}{+}\end{array}$ & $\begin{array}{l}Z \\
\dot{\infty} \\
\dot{m} \\
\stackrel{\sim}{y}\end{array}$ & $\begin{array}{l}z \\
\dot{N} \\
\vdots \\
\vdots\end{array}$ & $\begin{array}{l}z \\
\dot{2} \\
\stackrel{2}{\gamma}\end{array}$ & $\begin{array}{l}z \\
\dot{2} \\
\dot{y} \\
\dot{y}\end{array}$ & $\begin{array}{l}\text { Z } \\
\dot{+} \\
\stackrel{+}{\sigma}\end{array}$ & $\begin{array}{l}Z \\
\dot{ } \\
\stackrel{1}{0} \\
\stackrel{+}{7}\end{array}$ & $\begin{array}{l}Z \\
\dot{\infty} \\
\dot{m} \\
\stackrel{0}{\forall}\end{array}$ & $\begin{array}{l}Z \\
\dot{\infty} \\
m \\
\stackrel{y}{y}\end{array}$ & \\
\hline 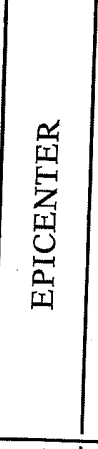 & 㒰 & 甹 & 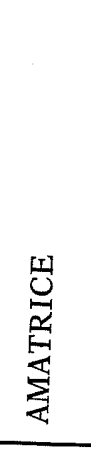 & 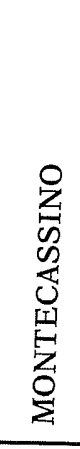 & 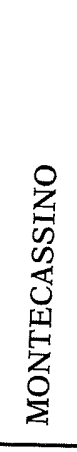 & 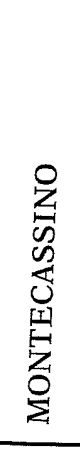 & 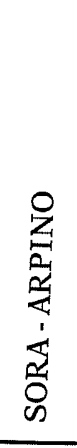 & 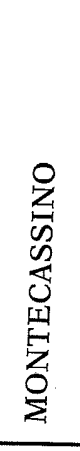 & 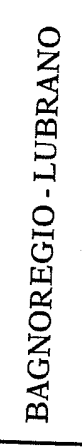 & 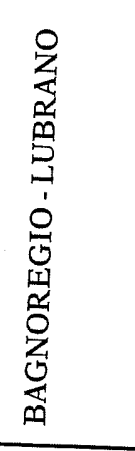 & \\
\hline $\begin{array}{r}1 \\
Z \\
0 \\
0 \\
\end{array}$ & 罕 & Z & Z & $\stackrel{\sim}{\sim}$ & $\Leftrightarrow$ & 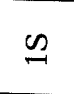 & $\mathscr{N}$ & 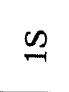 & Z & 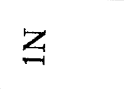 & \\
\hline 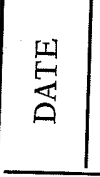 & $\frac{\substack{\text { bे } \\
\frac{1}{6} \\
\infty}}{\infty}$ & $\begin{array}{l}\frac{a}{0} \\
\frac{0}{1} \\
\frac{1}{d}\end{array}$ & $\begin{array}{l}\text { ò } \\
\frac{1}{0} \\
\frac{1}{1}\end{array}$ & \begin{tabular}{l}
\multirow{3}{*}{} \\
$\frac{1}{0}$ \\
\multirow{3}{J}{}
\end{tabular} & 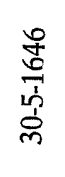 & 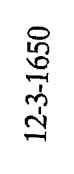 & $\begin{array}{l}\frac{4}{0} \\
\frac{1}{n} \\
\text { ஸे }\end{array}$ & $\begin{array}{l}\infty \\
0 \\
0 \\
\frac{1}{0} \\
0 \\
0\end{array}$ & 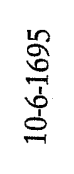 & \multirow{2}{*}{\multicolumn{2}{|c|}{ 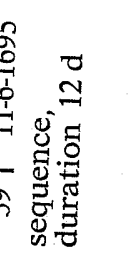 }} \\
\hline$z$ & 요 & in & กิ & กิ & in & in & ㄴํ & in & $\infty$ & & \\
\hline
\end{tabular}




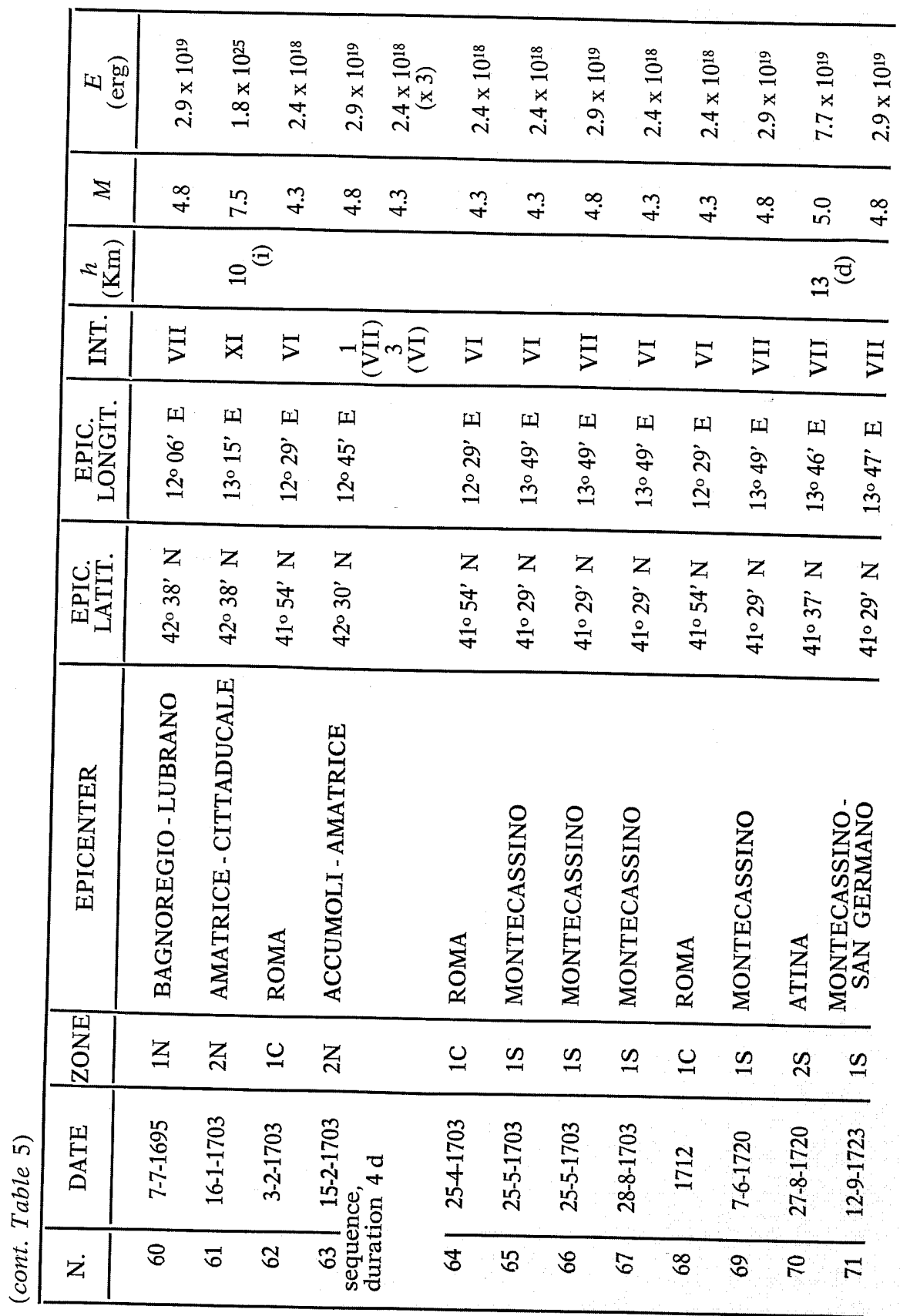




\begin{tabular}{|c|c|c|c|c|c|c|c|c|c|c|c|c|c|}
\hline 넝 & 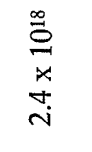 & $\begin{array}{l}\stackrel{\partial}{\sigma} \\
\dot{x} \\
\stackrel{a}{j}\end{array}$ & $\begin{array}{l}\stackrel{\infty}{\partial} \\
\underset{7}{x} \\
10 \\
0\end{array}$ & 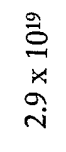 & $\begin{array}{l}\stackrel{\infty}{\circ} \\
\stackrel{x}{x} \\
\stackrel{+}{\sim}\end{array}$ & $\begin{array}{l}\stackrel{a}{0} \\
\dot{x} \\
\stackrel{-}{v}\end{array}$ & $\begin{array}{l}\stackrel{a}{\sigma} \\
\stackrel{x}{x} \\
\stackrel{a}{r}\end{array}$ & 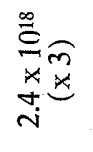 & $\begin{array}{l}\stackrel{a}{0} \\
\stackrel{x}{r} \\
\end{array}$ & 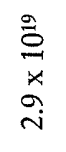 & $\begin{array}{l}\stackrel{\infty}{0} \\
x \\
x \\
0 \\
0\end{array}$ & $\begin{array}{l}\underset{0}{0} \\
x \\
\text { in } \\
\text { in }\end{array}$ & $\begin{array}{c}\underset{\delta}{0} \\
\dot{x} \\
m \\
\end{array}$ \\
\hline$\Sigma$ & $\stackrel{m}{+}$ & $\stackrel{\infty}{+}$ & $\stackrel{n}{\forall}$ & $\stackrel{\infty}{+}$ & $\stackrel{m}{+}$ & $\stackrel{\infty}{+}$ & $\stackrel{\infty}{+}$ & $\stackrel{m}{+}$ & in & $\stackrel{\infty}{\stackrel{\sim}{+}}$ & $\stackrel{n}{+}$ & $\stackrel{\circ}{\stackrel{\sim}{*}}$ & $\overrightarrow{\text { in }}$ \\
\hline$\approx \widehat{\Xi}$ & \multicolumn{5}{|c|}{ 0? } & \multicolumn{3}{|c|}{ 후 } & \multicolumn{2}{|c|}{$\underline{\tilde{\theta}}$} & \multicolumn{3}{|c|}{ 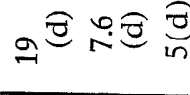 } \\
\hline 茕 & $\$$ & $\$$ & $\stackrel{5}{>}$ & $\stackrel{\ominus}{>}$ & 5 & $\stackrel{5}{>}$ & -13 & 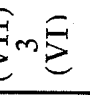 & $\Xi$ & 5 & 5 & 5 & $\Xi$ \\
\hline 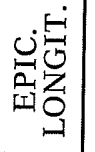 & 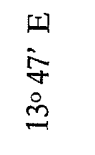 & 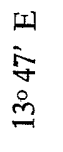 & $\begin{array}{l}\text { 되 } \\
\text { in } \\
\text { 号 } \\
\stackrel{\longrightarrow}{\longrightarrow}\end{array}$ & $\begin{array}{l}\text { II } \\
\text { in } \\
\stackrel{+}{0}\end{array}$ & $\begin{array}{l}\text { II } \\
\text { in } \\
\stackrel{+}{0} \\
\stackrel{2}{2}\end{array}$ & $\begin{array}{l}\text { II } \\
\dot{8} \\
\stackrel{8}{0} \\
\stackrel{1}{1}\end{array}$ & $\begin{array}{l}\text { 더 } \\
\dot{1} \\
\dot{+} \\
\stackrel{0}{-}\end{array}$ & & $\begin{array}{l}\text { 퍼 } \\
\dot{+} \\
\stackrel{+}{y}\end{array}$ & $\begin{array}{l}\text { 피 } \\
\dot{+} \\
\stackrel{+}{5} \\
\stackrel{y}{\square}\end{array}$ & $\begin{array}{l}\text { [I } \\
\dot{+} \\
\stackrel{+}{ \pm}\end{array}$ & 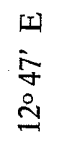 & $\begin{array}{l}\text { 디 } \\
\stackrel{\text { İ }}{\text { İ }}\end{array}$ \\
\hline 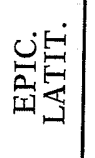 & $\begin{array}{l}z \\
\dot{2} \\
\stackrel{+}{\forall}\end{array}$ & 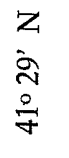 & $\begin{array}{l}z \\
\dot{0} \\
\dot{0} \\
\dot{\sigma}\end{array}$ & $\begin{array}{l}Z \\
\dot{2} \\
\dot{m} \\
\stackrel{+}{\sigma}\end{array}$ & $\begin{array}{l}Z \\
\dot{2} \\
\dot{m} \\
\dot{7}\end{array}$ & $\begin{array}{l}Z \\
\dot{\infty} \\
\dot{m} \\
\stackrel{\sim}{y}\end{array}$ & $\begin{array}{l}z \\
\text { ¿े } \\
\stackrel{\circ}{+}\end{array}$ & & $\begin{array}{l}Z \\
\dot{T} \\
\dot{\sigma} \\
\stackrel{\circ}{\sigma}\end{array}$ & $\begin{array}{l}z \\
\dot{0} \\
+ \\
\\
\end{array}$ & $\begin{array}{l}Z \\
\dot{Z} \\
\dot{+} \\
\stackrel{+}{+}\end{array}$ & $\begin{array}{l}Z \\
\dot{7} \\
\dot{7} \\
\dot{7}\end{array}$ & $\begin{array}{l}Z \\
\text { is } \\
\stackrel{+}{*} \\
\dot{\forall}\end{array}$ \\
\hline 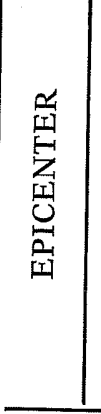 & 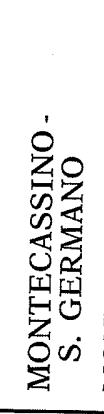 & 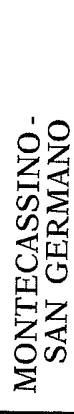 & 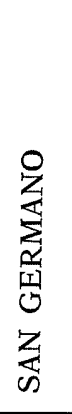 & 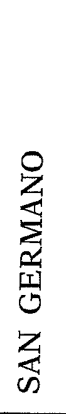 & 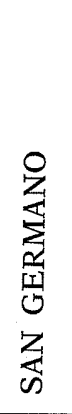 & 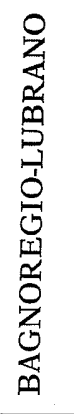 & 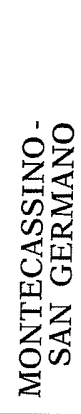 & & 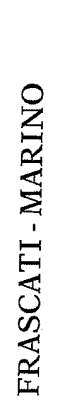 & 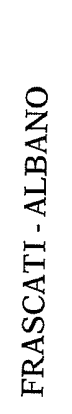 & 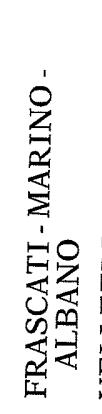 & 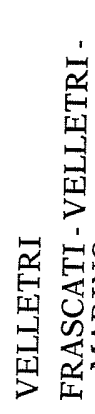 & \\
\hline $\begin{array}{l}1 \\
Z \\
\\
\end{array}$ & 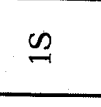 & 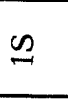 & $\mathscr{n}^{n}$ & $\stackrel{\sim}{\sim}$ & $\stackrel{\infty}{\infty}$ & ZZ & $\stackrel{\sim}{\sim}$ & & 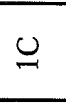 & 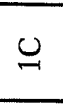 & $\stackrel{\cup}{\ddots}$ & $\cup$ & $\underline{U}$ \\
\hline 嵌 & $\begin{array}{l}\stackrel{2}{\mathbb{N}} \\
\frac{1}{0} \\
\frac{1}{\leftrightarrows}\end{array}$ & $\begin{array}{c}\stackrel{2}{\mathbb{N}} \\
\frac{1}{0} \\
\frac{1}{0} \\
\end{array}$ & $\frac{\vec{n}}{\overrightarrow{+}}$ & $\begin{array}{l}\vec{m} \\
\stackrel{7}{7} \\
\text { d }\end{array}$ & 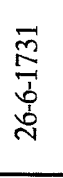 & $\stackrel{\infty}{\stackrel{m}{=}}$ & 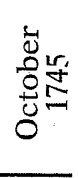 & 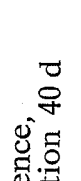 & $\begin{array}{l}\text { 京 } \\
\frac{1}{a} \\
\stackrel{1}{=}\end{array}$ & 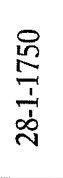 & $\frac{\stackrel{ }{n}}{\stackrel{\sim}{\sim}}$ & 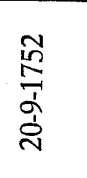 & $\begin{array}{l}\text { N } \\
\text { ล̄ } \\
\text { ò }\end{array}$ \\
\hline$z$ & 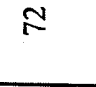 & $\cong$ & $\underset{N}{ \pm}$ & in & $\stackrel{P}{2}$ & $\approx$ & $\stackrel{\infty}{\sim}$ & 忌 & 9 & $\triangleright$ & $\bar{\infty}$ & ळ & ని \\
\hline
\end{tabular}




\begin{tabular}{|c|c|c|c|c|c|c|c|c|c|c|c|c|c|}
\hline 山्य & $\begin{array}{l}\stackrel{\partial}{0} \\
x \\
\dot{x} \\
\dot{y}\end{array}$ & 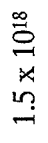 & 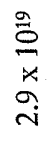 & 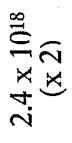 & 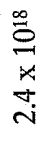 & $\begin{array}{l}\stackrel{\infty}{\sigma} \\
\stackrel{a}{*} \\
\stackrel{+}{\sim}\end{array}$ & $\begin{array}{l}\stackrel{\infty}{0} \\
\stackrel{0}{1} \\
\dot{x} \\
\stackrel{\sim}{d}\end{array}$ & $\begin{array}{l}\stackrel{a}{0} \\
\dot{x} \\
\mathfrak{\alpha}\end{array}$ & $\begin{array}{l}\infty \\
\stackrel{0}{\sigma} \\
\not{y} \\
\dot{v} \\
\dot{v}\end{array}$ & $\begin{array}{l}\stackrel{\infty}{o} \\
\stackrel{x}{x} \\
\stackrel{+}{j}\end{array}$ & $\begin{array}{l}\stackrel{\infty}{\sigma} \\
\qquad \\
\dot{\alpha} \\
\dot{v} \\
\sim\end{array}$ & 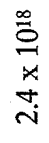 & $\begin{array}{l}\stackrel{\infty}{\circ} \\
\stackrel{x}{x} \\
\stackrel{+}{i}\end{array}$ \\
\hline$\Sigma$ & $\stackrel{\vartheta}{+}$ & $\stackrel{\sim}{\sim}$ & $\stackrel{\infty}{+}$ & $\stackrel{m}{*}$ & $\stackrel{m}{+}$ & $\ddot{q}$ & $\stackrel{m}{+}$ & $\stackrel{\infty}{+}$ & $\stackrel{m}{\forall}$ & $\stackrel{m}{\sigma}$ & $\stackrel{\dddot{q}}{\forall}$ & $\dddot{m}$ & $\dddot{q}$ \\
\hline$\approx \widehat{\Xi}$ & $\approx$ & & & & & & & & & & & & \\
\hline 旨 & 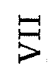 & 5 & & & 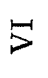 & 5 & 5 & $\$$ & 5 & 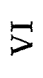 & 5 & 5 & 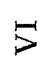 \\
\hline 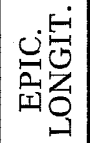 & $\begin{array}{l}\text { 되 } \\
\text { io } \\
\text { ㅁ } \\
\stackrel{\text { I }}{y}\end{array}$ & $\begin{array}{l}\text { 미 } \\
\check{0} \\
\stackrel{0}{ \pm}\end{array}$ & $\begin{array}{l}\text { 되 } \\
\text { 응 } \\
\stackrel{0}{=}\end{array}$ & & $\begin{array}{l}\text { II } \\
\text { in } \\
0 \\
\stackrel{0}{=}\end{array}$ & $\begin{array}{l}\text { 되 } \\
\text { 음 } \\
\stackrel{9}{9}\end{array}$ & \begin{tabular}{l} 
디 \\
\multirow{8}{+}{} \\
$\stackrel{8}{=}$
\end{tabular} & 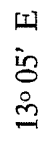 & $\begin{array}{l}\text { II } \\
\text { ò } \\
\text { o } \\
\stackrel{m}{\sim}\end{array}$ & $\begin{array}{l}\text { 되 } \\
\dot{2} \\
\dot{0} \\
\dot{0}\end{array}$ & $\begin{array}{l}\text { 디 } \\
\text { 음 } \\
\stackrel{0}{ป}\end{array}$ & $\begin{array}{l}\text { 디 } \\
\dot{7} \\
\stackrel{+}{+}\end{array}$ & $\begin{array}{l}\text { 되 } \\
\text { 우 } \\
\stackrel{ }{\Xi}\end{array}$ \\
\hline 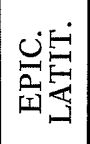 & $\begin{array}{l}z \\
\dot{\circ} \\
\text { in } \\
\stackrel{\circ}{7}\end{array}$ & 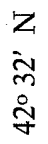 & 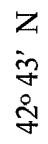 & & $\begin{array}{l}Z \\
\dot{0} \\
0 \\
\stackrel{7}{7}\end{array}$ & 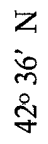 & 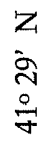 & $\begin{array}{l}\text { Z } \\
\text { ì } \\
\frac{0}{7}\end{array}$ & $\begin{array}{l}z \\
\grave{\nu} \\
\stackrel{0}{\sigma}\end{array}$ & $\begin{array}{l}Z \\
\dot{\gamma} \\
\dot{8} \\
\stackrel{\circ}{7}\end{array}$ & $\begin{array}{l}\text { Z } \\
\dot{0} \\
\text { 1 } \\
\frac{0}{8}\end{array}$ & $\begin{array}{l}z \\
\dot{0} \\
\dot{+} \\
\stackrel{0}{+}\end{array}$ & $\begin{array}{l}z \\
\dot{m} \\
\dot{q} \\
\stackrel{+}{y}\end{array}$ \\
\hline
\end{tabular}

\begin{tabular}{|c|c|c|c|c|c|c|c|c|c|c|c|c|}
\hline 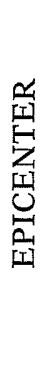 & 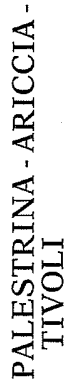 & 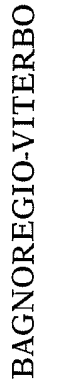 & 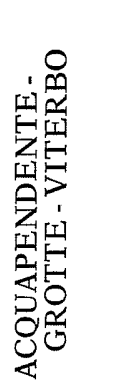 & $\begin{array}{l}\text { N1 } \\
\text { NT } \\
\text { N }\end{array}$ & 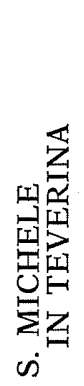 & 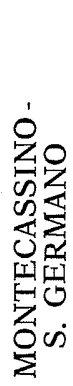 & 空 & 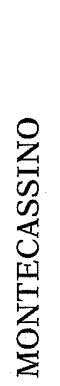 & 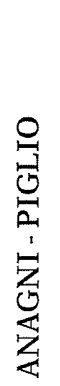 & 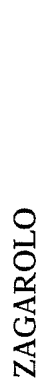 & 莣 & 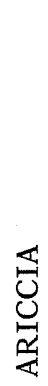 \\
\hline $\begin{array}{l}\text { 띰 } \\
\text { Z } \\
\text { 인 }\end{array}$ & $\cup$ & $\underline{Z}$ & ZZ & 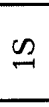 & 吕 & $\stackrel{\sim}{=}$ & $\stackrel{\sim}{\sim}$ & $\mathscr{D}^{+1}$ & $\stackrel{\sim}{\sim}$ & $\stackrel{\cup}{\smile}$ & 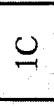 & $\stackrel{0}{\cup}$ \\
\hline 罩 & 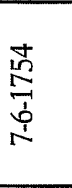 & 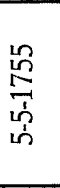 & 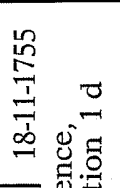 & $\frac{\stackrel{2}{2}}{\frac{1}{\sim}}$ & $\begin{array}{l}\frac{9}{1} \\
\stackrel{7}{0} \\
\vdots \\
7\end{array}$ & $\frac{9}{\frac{1}{5}}$ & $\stackrel{\text { : }}{\stackrel{2}{L}}$ & $\underset{\frac{D}{7}}{\frac{7}{7}}$ & 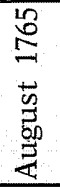 & 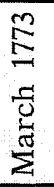 & 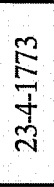 & 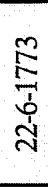 \\
\hline 2 & ஓ & $\stackrel{12}{\infty}$ & ๖ & 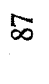 & $\infty$ & $\stackrel{\infty}{ }$ & 8 & Б & $\alpha$ & 2 & $\$$ & น \\
\hline
\end{tabular}




\begin{tabular}{|c|c|c|c|c|c|c|c|c|c|c|c|c|c|}
\hline 四曽 & $\begin{array}{l}\infty \\
\text { 을 } \\
x \\
n \\
n \\
0\end{array}$ & $\begin{array}{l}\stackrel{9}{\partial} \\
\stackrel{x}{x} \\
\stackrel{\jmath}{v}\end{array}$ & 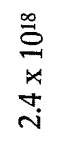 & $\begin{array}{l}\stackrel{0}{0} \\
x \\
x \\
0 \\
r\end{array}$ & $\begin{array}{l}\stackrel{\infty}{\Xi} \\
\stackrel{x}{x} \\
\stackrel{+}{J}\end{array}$ & $\begin{array}{l}\stackrel{\infty}{\circ} \\
\stackrel{x}{x} \\
\stackrel{ \pm}{\sim}\end{array}$ & $\begin{array}{l}\stackrel{a}{\partial} \\
\stackrel{0}{x} \\
x \\
\infty \\
-i\end{array}$ & $\begin{array}{l}\stackrel{a}{\sigma} \\
\dot{y} \\
a \\
i\end{array}$ & 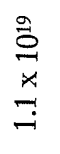 & $\begin{array}{l}\stackrel{9}{\sigma} \\
\not \\
x \\
\infty \\
-1\end{array}$ & $\begin{array}{l}\stackrel{8}{0} \\
\not x \\
b \\
\dot{0} \\
\text { in }\end{array}$ & $\begin{array}{l}\stackrel{n}{0} \\
\underset{x}{*} \\
\stackrel{+}{v}\end{array}$ & $\begin{array}{l}\stackrel{\infty}{0} \\
x \\
n \\
0 \\
0\end{array}$ \\
\hline$\Xi$ & $\stackrel{\ln }{*}$ & $\stackrel{\infty}{+}$ & $\dddot{r}$ & $\stackrel{0}{\dot{m}}$ & $\stackrel{n}{+}$ & $\stackrel{m}{\sigma}$ & $\underset{+}{ }$ & $\stackrel{\infty}{+}$ & $\stackrel{\circ}{+}$ & $\stackrel{\nabla}{\forall}$ & $\overrightarrow{i n}$ & 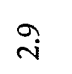 & in \\
\hline 졸 & \multicolumn{6}{|c|}{ 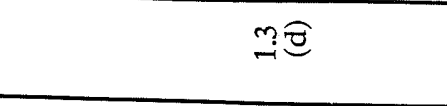 } & \multicolumn{2}{|c|}{$\infty$ త్ర } & \multicolumn{5}{|c|}{ 유 } \\
\hline$\underset{Z}{Z}$ & $\dot{5}$ & 5 & 5 & 5 & 5 & $\$$ & $\stackrel{5}{P}$ & 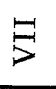 & 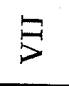 & 5 & $\stackrel{\Xi}{\lessgtr}$ & $\Xi$ & 占卢 \\
\hline 旨 & $\begin{array}{l}\text { 피 } \\
\text { ஸे } \\
\text { ஸे }\end{array}$ & $\begin{array}{l}\text { II } \\
\dot{m} \\
\stackrel{m}{m} \\
\stackrel{m}{m}\end{array}$ & $\begin{array}{l}\text { 피 } \\
\dot{+} \\
\stackrel{ }{\nearrow}\end{array}$ & 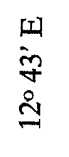 & $\begin{array}{l}\text { 피 } \\
\text { ஸे } \\
\stackrel{0}{=} \\
\Rightarrow\end{array}$ & $\begin{array}{l}\text { 되 } \\
\dot{+} \\
\stackrel{+}{\square}\end{array}$ & $\begin{array}{l}\text { ㅍ } \\
\text { în } \\
\text { ปิ }\end{array}$ & 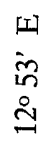 & 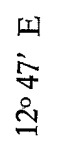 & $\begin{array}{l}\text { 되 } \\
\dot{0} \\
10 \\
\stackrel{0}{n}\end{array}$ & 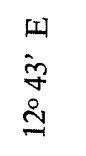 & $\begin{array}{l}\text { II } \\
\dot{J} \\
\stackrel{+}{\Xi}\end{array}$ & $\begin{array}{l}\text { 되 } \\
\dot{+} \\
\stackrel{+}{0} \\
\stackrel{ }{-}\end{array}$ \\
\hline 岕舅 & $\begin{array}{l}Z \\
\dot{Z} \\
\stackrel{\sim}{\circ} \\
\stackrel{+}{\forall}\end{array}$ & 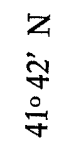 & $\begin{array}{l}Z \\
i \\
\dddot{q} \\
\stackrel{7}{q}\end{array}$ & $\begin{array}{l}z \\
\dot{a} \\
\dot{\sigma} \\
\dot{\sigma}\end{array}$ & $\begin{array}{l}z \\
\dot{z} \\
\dot{m} \\
\stackrel{y}{y}\end{array}$ & $\begin{array}{l}z \\
\dot{y} \\
\stackrel{+}{q}\end{array}$ & $\begin{array}{l}z \\
\text { in } \\
\text { in } \\
\stackrel{0}{+} \\
\dot{y}\end{array}$ & $\begin{array}{l}z \\
\text { in } \\
\text { in } \\
\stackrel{2}{7}\end{array}$ & $\begin{array}{l}Z \\
\dot{Z} \\
\dot{7} \\
\stackrel{0}{\sigma}\end{array}$ & $\begin{array}{l}z \\
\dot{2} \\
\dot{2} \\
\stackrel{+}{+}\end{array}$ & $\begin{array}{l}Z \\
\dot{J} \\
\dot{+} \\
\dot{y}\end{array}$ & $\begin{array}{l}Z \\
\dot{\infty} \\
\dot{+} \\
\dot{+}\end{array}$ & $\begin{array}{l}z \\
\text { in } \\
\dot{y} \\
\dot{y}\end{array}$ \\
\hline 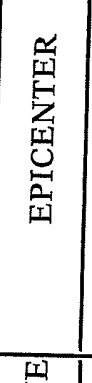 & $\underset{\sigma}{E}$ & 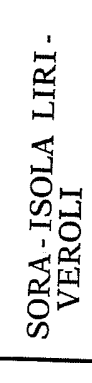 & 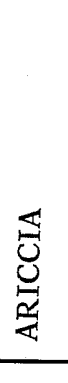 & 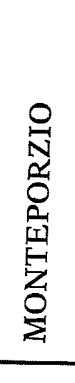 & $\begin{array}{l}\sum_{1} \\
0 \\
0 \\
0 \\
0 \\
0\end{array}$ & 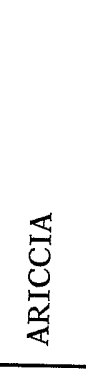 & 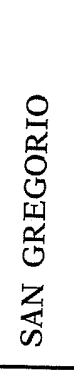 & 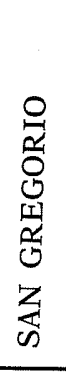 & 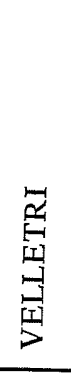 & 总 & 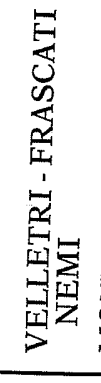 & 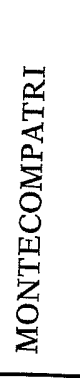 & 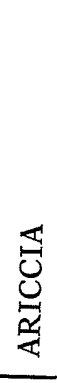 \\
\hline Z & 录 & $\stackrel{\sim}{\sim}$ & $\underline{U}$ & $\underset{-1}{U}$ & Z & $\stackrel{\cup}{\smile}$ & 吕 & Z & $\stackrel{\cup}{0}$ & 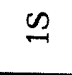 & $\underset{-}{\mathcal{U}}$ & 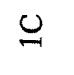 & $\bigcup$ \\
\hline 乩 & $\frac{\mathfrak{N}}{\stackrel{1}{2}}$ & $\underset{\Xi}{E}$ & 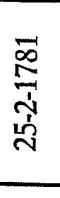 & 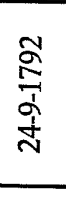 & $\frac{2}{\frac{2}{1}}$ & 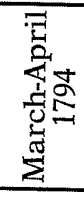 & 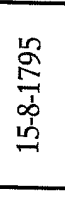 & 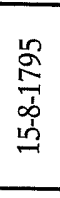 & 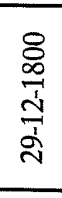 & $\begin{array}{l}0 \\
\infty \\
\frac{0}{1} \\
\frac{1}{\pi}\end{array}$ & 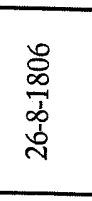 & 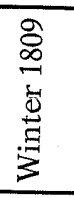 & $\underset{\infty}{0}$ \\
\hline$\ddot{z}$ & 20 & Б̃ & ঞ̊ & g & § & $\overline{0}$ & $\underset{O}{O}$ & $\stackrel{\approx}{\sigma}$ & む & $\stackrel{2}{\circ}$ & ஜำ & 음 & $\stackrel{\infty}{\circ}$ \\
\hline
\end{tabular}


PHENOMENOLOGIC ASPECTS OF SEISMICITY IN LATIUM

\begin{tabular}{|c|c|c|c|c|c|c|c|c|c|c|c|}
\hline 四总 & $\begin{array}{l}\stackrel{0}{0} \\
\stackrel{5}{x} \\
\stackrel{\infty}{+}\end{array}$ & 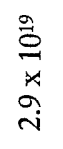 & 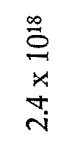 & $\begin{array}{l}\stackrel{\partial}{\partial} \\
\bar{x} \\
\hat{i}\end{array}$ & 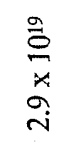 & 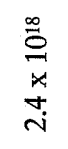 & $\begin{array}{l}\stackrel{\infty}{\leftrightarrows} \\
\stackrel{x}{\alpha} \\
\stackrel{+}{i}\end{array}$ & $\begin{array}{l}\stackrel{\infty}{\Xi} \\
\stackrel{x}{x} \\
\stackrel{+}{v}\end{array}$ & 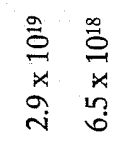 & 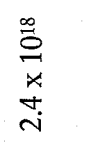 & 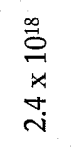 \\
\hline$\Sigma$ & $\stackrel{\sim}{+}$ & $\stackrel{\infty}{+}$ & $\stackrel{m}{+}$ & $\stackrel{\infty}{+}$ & $\stackrel{\infty}{+}$ & $\stackrel{m}{*}$ & $\stackrel{m}{+}$ & $\stackrel{m}{*}$ & 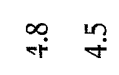 & $\stackrel{m}{+}$ & $\stackrel{m}{+}$ \\
\hline$\therefore \widehat{g}$ & \multicolumn{6}{|l|}{$\stackrel{0}{0}$} & \multicolumn{5}{|c|}{$+\Theta$} \\
\hline$\dot{\mathrm{z}}$ & s. & & 5 & 5 & 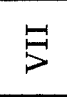 & 5 & 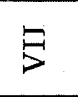 & 5 & $-\widehat{E}$ & $\bar{E}-\widehat{E}$ & 5 \\
\hline 易 & 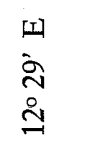 & $\begin{array}{l}\text { 피 } \\
\grave{\grave{े}} \\
\grave{\Xi}\end{array}$ & $\begin{array}{l}\text { 떠 } \\
\dot{+} \\
\stackrel{+}{\beth}\end{array}$ & $\begin{array}{l}\text { 미 } \\
\text { io } \\
\stackrel{0}{0} \\
=\end{array}$ & 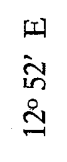 & $\begin{array}{l}\text { ㅍ } \\
\dot{\sigma} \\
\text { స్ }\end{array}$ & $\begin{array}{l}\text { ㅁ } \\
\dot{\sigma} \\
\stackrel{\Xi}{\Xi}\end{array}$ & $\begin{array}{l}\text { ㅁ } \\
\dot{y} \\
\stackrel{+}{\check{Z}}\end{array}$ & $\begin{array}{l}\text { 띠 } \\
\dot{\sigma} \\
\stackrel{9}{9}\end{array}$ & & $\begin{array}{l}\text { 디 } \\
\dot{+} \\
\stackrel{5}{2}\end{array}$ \\
\hline 总窇 & $\begin{array}{l}Z \\
\dot{H} \\
\dot{0} \\
\dot{y}\end{array}$ & $\begin{array}{l}z \\
\dot{i} \\
0 \\
\dot{0} \\
\forall\end{array}$ & $\begin{array}{l}z \\
j \\
j \\
0 \\
\dot{y}\end{array}$ & $\begin{array}{l}z \\
\text { in } \\
\stackrel{8}{q} \\
y\end{array}$ & 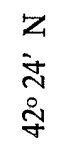 & $\begin{array}{l}z \\
\dot{z} \\
\dot{q} \\
\dot{0} \\
\dot{y}\end{array}$ & $\begin{array}{l}z \\
\dot{z} \\
\dot{q} \\
\dot{y}\end{array}$ & $\begin{array}{l}z \\
\text { in } \\
+ \\
\frac{0}{7}\end{array}$ & $\begin{array}{l}z \\
\dot{N} \\
\dot{0} \\
\dot{\nabla}\end{array}$ & & $\begin{array}{l}z_{1} \\
\dot{i} \\
\dot{a} \\
\dot{y}\end{array}$ \\
\hline 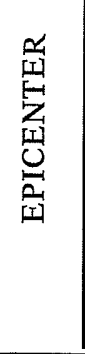 & 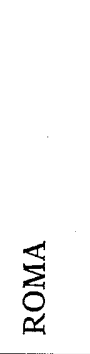 & 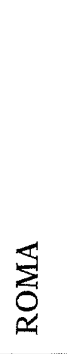 & 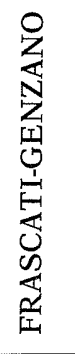 & 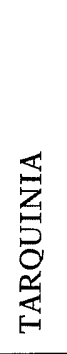 & 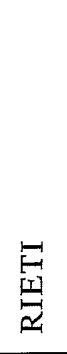 & 是 & 足 & 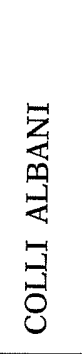 & 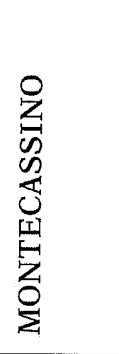 & & 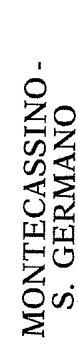 \\
\hline $\begin{array}{l}\text { II } \\
\text { Z } \\
\text { O } \\
\end{array}$ & $\underline{U}$ & 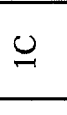 & $\stackrel{0}{\cup}$ & Z & 学 & $\stackrel{\cup}{\cup}$ & 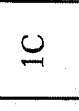 & 0 & $\mathscr{\omega}^{n}$ & & $\omega^{4}$ \\
\hline 奌 & $\begin{array}{l}\overrightarrow{0} \\
\stackrel{1}{\overrightarrow{1}} \\
\infty\end{array}$ & 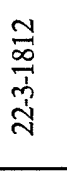 & 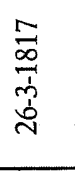 & 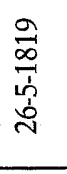 & 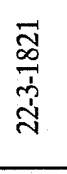 & 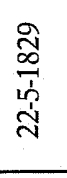 & 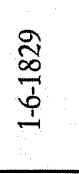 & 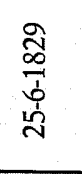 & 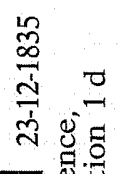 & & $\begin{array}{c}\infty \\
\infty \\
\stackrel{D}{d}\end{array}$ \\
\hline$r_{1}$ & gे & $\stackrel{ }{ }$ & $\exists$ & $\cong$ & $\stackrel{m}{=}$ & $\Xi$ & $\stackrel{n}{=}$ & $\cong$ & 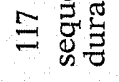 & & $\stackrel{\infty}{=}$ \\
\hline
\end{tabular}




\begin{tabular}{|c|c|c|c|c|c|c|c|c|c|c|c|c|c|}
\hline 四兽 & 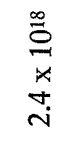 & $\begin{array}{l}\stackrel{a}{0} \\
\\
\stackrel{y}{v}\end{array}$ & $\begin{array}{l}\hat{\sigma} \\
\stackrel{1}{x} \\
\vec{a}\end{array}$ & $\begin{array}{l}\infty \\
\stackrel{\infty}{0} \\
\dot{x} \\
\stackrel{\sim}{\sim}\end{array}$ & $\begin{array}{l}0 \\
\stackrel{0}{0} \\
\\
\infty \\
\end{array}$ & 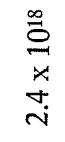 & $\begin{array}{l}\stackrel{\Xi}{0} \\
\stackrel{1}{4} \\
m \\
-\end{array}$ & 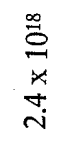 & $\begin{array}{c}0 \\
0 \\
x \\
x \\
n \\
0\end{array}$ & $\begin{array}{l}0 \\
\stackrel{0}{0} \\
x \\
\dot{x} \\
\dot{N}\end{array}$ & 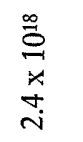 & $\begin{array}{l}\stackrel{ \pm}{0} \\
\stackrel{0}{x} \\
\dot{a} \\
i n\end{array}$ & $\frac{5}{0}$ \\
\hline$\Xi$ & $\stackrel{\Re}{\forall}$ & $\stackrel{\infty}{+}$ & $\widetilde{7}$ & $\stackrel{m}{*}$ & $\stackrel{\dot{m}}{\stackrel{*}{*}}$ & $\ddot{q}$ & $\stackrel{r}{\sim}$ & $\ddot{q}$ & $\stackrel{20}{7}$ & $\ddot{q}$ & $\stackrel{m}{+}$ & $\stackrel{\circ}{\sim}$ & $\ddot{+}$ \\
\hline 촗 & \multicolumn{4}{|c|}{ m) } & \multicolumn{4}{|c|}{ m } & \multicolumn{5}{|c|}{$\stackrel{\infty}{\circ} 0.0$} \\
\hline 占 & 5 & $\stackrel{9}{S}$ & $\stackrel{9}{\triangleright}$ & 5 & 5 & 㟔口 & 5 & 5 & $\stackrel{2}{>}$ & 5 & 5 & 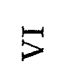 & 5 \\
\hline 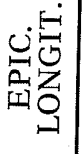 & 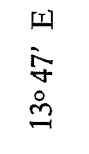 & 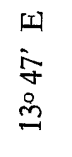 & 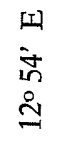 & 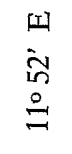 & $\begin{array}{l}\text { 되 } \\
\dot{F} \\
\stackrel{+}{ \pm}\end{array}$ & $\begin{array}{l}\text { म } \\
\overline{7} \\
\stackrel{7}{y}\end{array}$ & 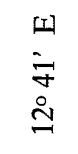 & $\begin{array}{l}\text { 디 } \\
\tilde{I} \\
\stackrel{0}{\circ} \\
\stackrel{0}{n}\end{array}$ & $\begin{array}{l}\text { 뙤 } \\
\dot{3} \\
\stackrel{+}{\Xi}\end{array}$ & $\begin{array}{l}\text { 띠 } \\
\hat{i} \\
\stackrel{+}{+} \\
\stackrel{0}{-}\end{array}$ & $\begin{array}{l}\text { 디 } \\
\dot{\hat{\gamma}} \\
\stackrel{0}{-}\end{array}$ & $\begin{array}{l}\text { 디 } \\
\dot{\sigma} \\
\dot{q} \\
\stackrel{-}{7}\end{array}$ & 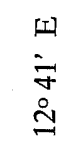 \\
\hline 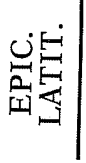 & $\begin{array}{l}z \\
\dot{\jmath} \\
\vdots \\
\dot{\sigma}\end{array}$ & $\begin{array}{l}Z \\
\text { Zे } \\
\dot{N} \\
\stackrel{+}{+}\end{array}$ & $\begin{array}{l}z \\
\dot{0} \\
10 \\
0 \\
\dot{7}\end{array}$ & $\begin{array}{l}z \\
\dot{+} \\
\dot{+} \\
\stackrel{9}{y}\end{array}$ & $\begin{array}{l}z \\
\dot{a} \\
\dot{+} \\
\stackrel{\circ}{+}\end{array}$ & $\begin{array}{l}Z \\
\dot{q} \\
\dot{+} \\
\dot{8}\end{array}$ & $\begin{array}{l}Z \\
\dot{a} \\
\dot{+} \\
\dot{7}\end{array}$ & $\begin{array}{l}Z \\
\dot{0} \\
\infty \\
\stackrel{y}{y}\end{array}$ & $\begin{array}{l}z \\
\dot{\sigma} \\
\dot{\sigma} \\
\dot{\sigma}\end{array}$ & $\begin{array}{l}z \\
\dot{N} \\
\dot{\nabla} \\
\dot{y}\end{array}$ & $\begin{array}{l}z \\
\dot{N} \\
\circ \\
\dot{+}\end{array}$ & $\begin{array}{l}z \\
\dot{⿰} \\
\circ \\
\stackrel{+}{+}\end{array}$ & 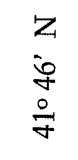 \\
\hline 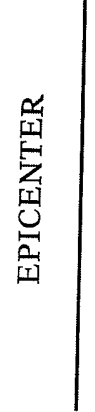 & 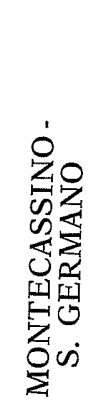 & 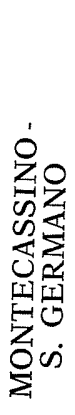 & 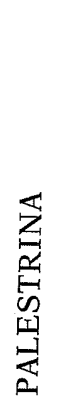 & 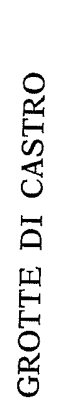 & 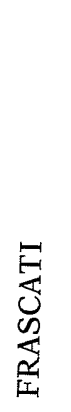 & 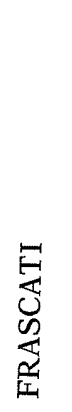 & 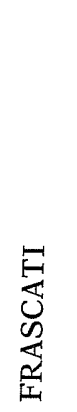 & 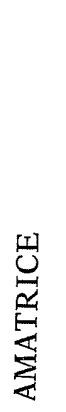 & 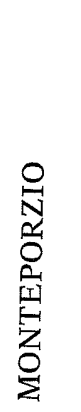 & 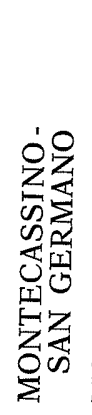 & 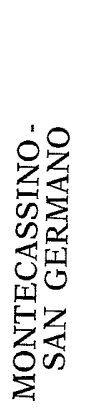 & 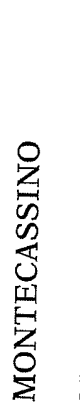 & 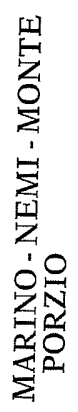 \\
\hline $\begin{array}{l}\frac{1}{Z} \\
\text { O } \\
\end{array}$ & $\stackrel{\vec{H}}{\sim}$ & $\stackrel{H}{\rightarrow}$ & $\cup$ & 恣 & 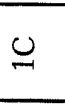 & 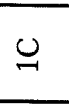 & $\cup$ & 栄 & $\stackrel{0}{0}$ & $\mathscr{\omega}^{-1}$ & $\stackrel{n}{n}$ & $\omega^{2}$ & $\stackrel{\cup}{\sim}$ \\
\hline 岌 & 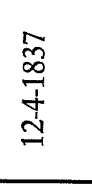 & $\begin{array}{l}\hat{2} \\
\frac{\infty}{1} \\
\frac{1}{ \pm}\end{array}$ & $\begin{array}{l}\frac{J}{ \pm} \\
\frac{1}{1} \\
\pm \\
\end{array}$ & 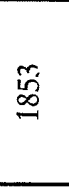 & 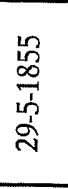 & 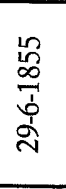 & 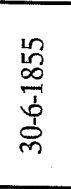 & 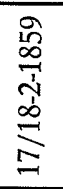 & 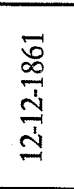 & 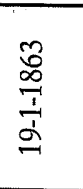 & $\begin{array}{l}m \\
\infty \\
\frac{1}{1} \\
\stackrel{d}{N}\end{array}$ & 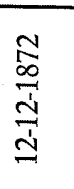 & $\frac{m}{\frac{m}{1}}$ \\
\hline$z$ & $\triangleq$ & ્ㅗ & $\widetilde{\Xi}$ & 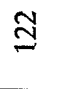 & $\stackrel{\dddot{\Xi}}{\exists}$ & $\stackrel{士}{\Xi}$ & $\stackrel{\mathscr{I}}{=}$ & $\underset{\sim}{\stackrel{2}{*}}$ & $\widehat{\Xi}$ & $\stackrel{\infty}{\underset{\sim}{*}}$ & ఫิ & లి & $\stackrel{m}{\sim}$ \\
\hline
\end{tabular}




\begin{tabular}{|c|c|c|c|c|c|c|c|c|c|c|c|c|c|}
\hline 山总 & 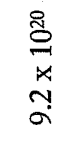 & 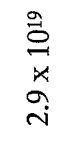 & $\begin{array}{l}\vec{\delta} \\
\ddot{x} \\
b \\
b\end{array}$ & $\begin{array}{l}\stackrel{\infty}{0} \\
\qquad x \\
x \\
0 \\
0\end{array}$ & 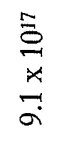 & 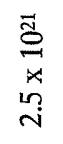 & 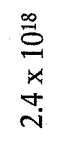 & 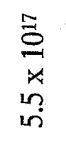 & 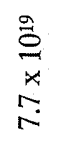 & $\begin{array}{l}\stackrel{\infty}{0} \\
\not{x} \\
\stackrel{+}{*}\end{array}$ & $\begin{array}{l}\infty \\
0 \\
\not x \\
i n \\
0\end{array}$ & 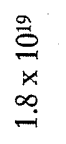 & 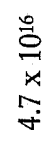 \\
\hline$\Sigma$ & in & $\stackrel{\infty}{\dot{\forall}}$ & in & $\stackrel{10}{+}$ & $\dddot{+}$ & in & $\stackrel{m}{7}$ & $\stackrel{\circ}{\dot{\sigma}}$ & 오 & $\dddot{m}$ & $\stackrel{2 n}{7}$ & $\dot{F}$ & $m$ \\
\hline 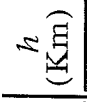 & \multicolumn{2}{|c|}{$\exists$} & $\bar{v}$ & \multicolumn{7}{|c|}{ 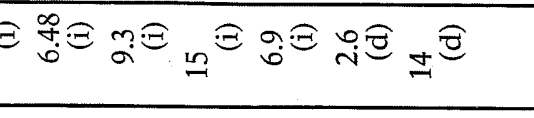 } & \multicolumn{3}{|c|}{ 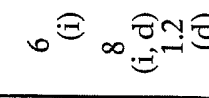 } \\
\hline 艺 & $\Xi$ & $\stackrel{F}{\lessgtr}$ & $\underset{⿱ 乛}{\rightleftharpoons}$ & $\stackrel{5}{>}$ & 5 & 导 & 龒 & $\stackrel{9}{>}$ & $\exists$ & 5 & 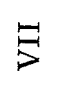 & 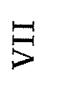 & $\Xi$ \\
\hline 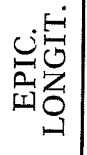 & $\begin{array}{l}\text { 되 } \\
\dot{+} \\
\dot{+} \\
\stackrel{0}{-}\end{array}$ & $\begin{array}{l}\text { ㅍ } \\
\dot{0} \\
\ddot{m} \\
\dot{m}\end{array}$ & $\begin{array}{l}\text { I } \\
\dot{\circ} \\
i n \\
\stackrel{0}{2}\end{array}$ & $\begin{array}{l}\text { ㅍ } \\
\dot{\dot{H}} \\
\stackrel{0}{ป}\end{array}$ & 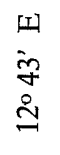 & $\begin{array}{l}\text { II } \\
\text { in } \\
\stackrel{0}{2} \\
\stackrel{2}{=}\end{array}$ & $\begin{array}{l}\text { 디 } \\
\text { ㅎㅇ } \\
\stackrel{0}{I}\end{array}$ & $\begin{array}{l}\text { 되 } \\
\dot{8} \\
\text { ㅇ } \\
\text { ㅇ }\end{array}$ & $\begin{array}{l}\text { 山 } \\
\text { in } \\
\text { in } \\
\stackrel{=}{=}\end{array}$ & 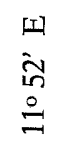 & $\begin{array}{l}\text { II } \\
\stackrel{\text { I }}{\leftrightarrows} \\
\stackrel{\Xi}{\leftrightarrows}\end{array}$ & $\begin{array}{l}\text { 피 } \\
\text { 호 } \\
\stackrel{\text { ㅇ }}{二}\end{array}$ & $\begin{array}{l}\text { 되 } \\
\dot{ } \\
\stackrel{+}{0} \\
\stackrel{0}{\sim}\end{array}$ \\
\hline 总曷 & $\begin{array}{l}z \\
\dot{q} \\
\dot{+} \\
\dot{y}\end{array}$ & 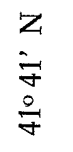 & 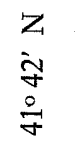 & $\begin{array}{l}Z \\
\dot{0} \\
10 \\
0 \\
\dot{7}\end{array}$ & $\begin{array}{l}z \\
\dot{0} \\
\dot{+} \\
\dot{y}\end{array}$ & $\begin{array}{l}Z \\
\dddot{Y} \\
\frac{0}{7}\end{array}$ & $\begin{array}{l}Z \\
\infty \\
\infty \\
\stackrel{7}{+}\end{array}$ & $\begin{array}{l}Z \\
\dot{j} \\
\stackrel{\sim}{\dot{y}}\end{array}$ & 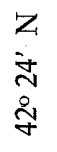 & $\begin{array}{l}z \\
\dot{m} \\
\stackrel{y}{y}\end{array}$ & $\begin{array}{l}Z \\
\dot{\infty} \\
\stackrel{+}{+} \\
\stackrel{0}{+}\end{array}$ & $\begin{array}{l}Z \\
i ̄ \\
m \\
\stackrel{y}{y}\end{array}$ & $\begin{array}{l}Z \\
i 3 \\
\dot{0} \\
\stackrel{0}{7}\end{array}$ \\
\hline 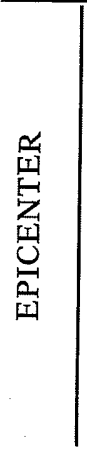 & 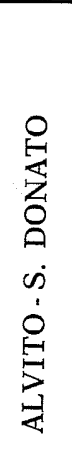 & 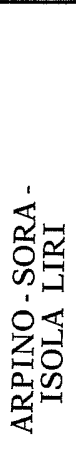 & 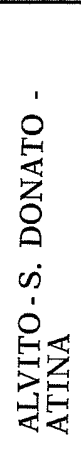 & 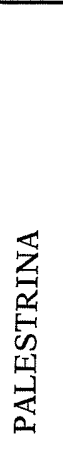 & 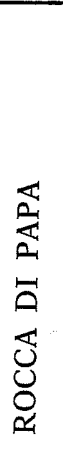 & $\begin{array}{l}3 \\
0 \\
\frac{1}{21} \\
>\end{array}$ & 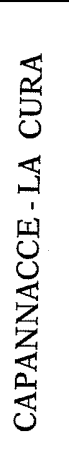 & 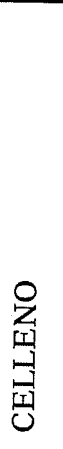 & 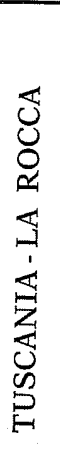 & 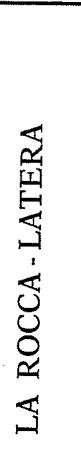 & 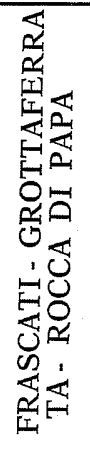 & 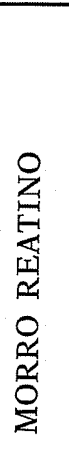 & 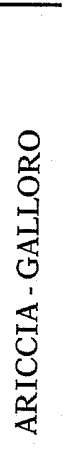 \\
\hline 至 & $\stackrel{\sim}{\sim}$ & PS & $\stackrel{\sim}{\sim}$ & $\underline{U}$ & $\underline{U}$ & $\stackrel{\sim}{N}$ & 朰 & Z Z & $\underline{G}$ & $\approx$ & $\simeq$ & 出 & $=$ \\
\hline 岳 & 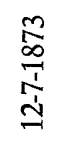 & 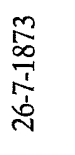 & 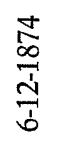 & 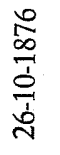 & $\begin{array}{l}\mathbb{N} \\
\infty \\
0 \\
0 \\
0 \\
0\end{array}$ & $\frac{N}{\infty}$ & 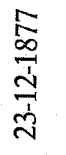 & 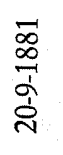 & $\stackrel{\infty}{a}$ & 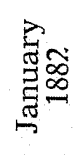 & $\frac{\infty}{\frac{\infty}{1}}$ & 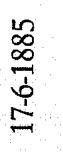 & $\frac{\infty}{\frac{1}{1}}$ \\
\hline$z$ & $\stackrel{\widetilde{2}}{2}$ & - & $\rightarrow$ & - & 2 & - & 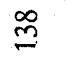 & - & $\simeq$ & - & $\triangle$ & \pm & \pm \\
\hline
\end{tabular}




\begin{tabular}{|c|c|c|c|c|c|c|c|c|c|c|c|c|}
\hline 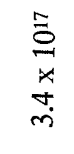 & 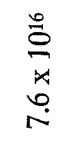 & $\begin{array}{l}\stackrel{\infty}{0} \\
\stackrel{\sim}{x} \\
\stackrel{+}{\sim}\end{array}$ & $\begin{array}{l}\stackrel{\infty}{0} \\
\underset{x}{+} \\
\stackrel{+}{i}\end{array}$ & 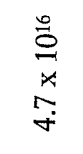 & 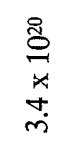 & 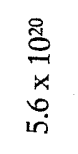 & 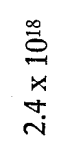 & $\begin{array}{l}\stackrel{\infty}{\sigma} \\
\underset{x}{*} \\
\stackrel{x}{n} \\
-\end{array}$ & 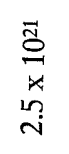 & 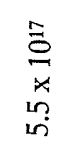 & 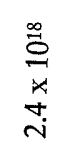 & $\begin{array}{l}\underset{\delta}{0} \\
\dot{x} \\
\sim \\
\sigma\end{array}$ \\
\hline$\stackrel{و}{m}$ & $\ddot{m}$ & $\dddot{m}$ & $\ddot{m}$ & $\stackrel{n}{n}$ & $\ddot{m}$ & i் & $\stackrel{m}{+}$ & $\underset{\forall}{\Upsilon}$ & in & $\stackrel{\circ}{+}$ & $\ddot{q}$ & $\begin{array}{l}\text { in } \\
\text { ir }\end{array}$ \\
\hline \multicolumn{3}{|c|}{ 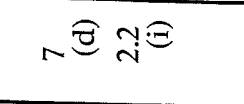 } & \multicolumn{5}{|c|}{ 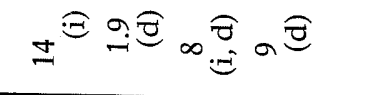 } & \multicolumn{4}{|c|}{ 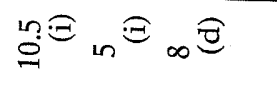 } & $\stackrel{\varrho}{=}$ \\
\hline 5 & $\stackrel{\text { 号 }}{\circ}$ & 5 & 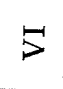 & 穴 & $\Xi$ & 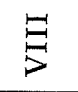 & 5 & $\stackrel{5}{>}$ & 局 & $\$$ & 5 & $\Xi$ \\
\hline $\begin{array}{l}\text { 미 } \\
\dot{\sim} \\
\stackrel{\sim}{ } \\
\stackrel{0}{-}\end{array}$ & $\begin{array}{l}\text { 되 } \\
\text { î } \\
\stackrel{\circ}{\Xi}\end{array}$ & $\begin{array}{l}\text { 띠 } \\
\text { ஸ̃ } \\
\stackrel{0}{\Xi}\end{array}$ & 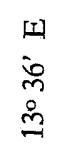 & $\begin{array}{l}\text { 띠 } \\
\dot{\sigma} \\
\stackrel{+}{0}\end{array}$ & $\begin{array}{l}\text { 되 } \\
\text { ั } \\
\stackrel{\text { I }}{\Xi}\end{array}$ & 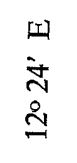 & $\begin{array}{l}\text { II } \\
\dot{Z} \\
\stackrel{\Delta}{\Xi}\end{array}$ & $\begin{array}{l}\text { II } \\
\dot{\vec{F}} \\
\cong\end{array}$ & $\begin{array}{l}\text { 피 } \\
\text { ஸे } \\
\text { ஸे }\end{array}$ & $\begin{array}{l}\text { 되 } \\
\text { in } \\
\text { in } \\
\stackrel{\Xi}{I}\end{array}$ & $\begin{array}{l}\text { 디 } \\
\text { 초 } \\
\stackrel{\text { I }}{ }\end{array}$ & $\begin{array}{l}\text { 되 } \\
\dot{8} \\
\stackrel{+}{0} \\
\stackrel{ }{\Xi}\end{array}$ \\
\hline $\begin{array}{l}Z \\
\dot{Z} \\
\dot{m} \\
o \\
\dot{\sigma}\end{array}$ & $\begin{array}{l}z \\
\dot{q} \\
\stackrel{+}{\stackrel{y}{+}}\end{array}$ & $\begin{array}{l}Z \\
\dot{+} \\
\dot{+} \\
\stackrel{\sim}{+}\end{array}$ & $\begin{array}{l}Z \\
\dot{m} \\
\dot{q} \\
\dot{g}\end{array}$ & $\begin{array}{l}Z \\
\text { Z } \\
\grave{2} \\
\stackrel{\circ}{\forall}\end{array}$ & $\begin{array}{l}Z \\
\dot{Z} \\
\dot{+} \\
\stackrel{0}{+}\end{array}$ & $\begin{array}{l}Z \\
\text { Z } \\
\text { î } \\
\stackrel{2}{+}\end{array}$ & $\begin{array}{l}z \\
i \bar{n} \\
i n \\
\dot{+}\end{array}$ & $\begin{array}{l}Z \\
\tilde{F} \\
\dot{\sigma} \\
\dot{\sigma}\end{array}$ & $\begin{array}{l}Z \\
\dot{z} \\
\dot{\sim} \\
\stackrel{y}{+}\end{array}$ & $\begin{array}{l}Z \\
\dot{\tilde{N}} \\
\stackrel{\sim}{\tilde{y}} \\
\dot{y}\end{array}$ & $\begin{array}{l}\text { Z } \\
\dot{\sim} \\
\stackrel{+}{Y} \\
\stackrel{+}{Y}\end{array}$ & $\begin{array}{l}Z \\
Z \\
\dot{\gamma} \\
\stackrel{\circ}{\gamma}\end{array}$ \\
\hline 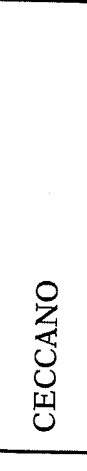 & 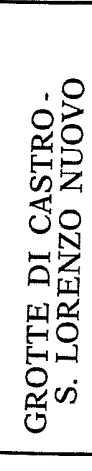 & 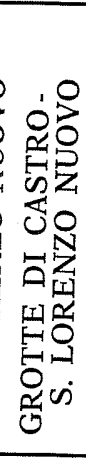 & 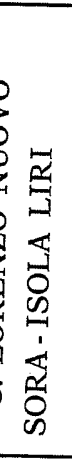 & 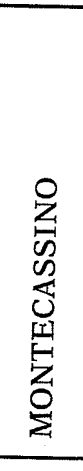 & 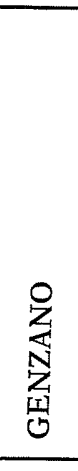 & 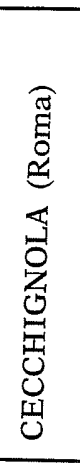 & 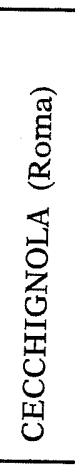 & 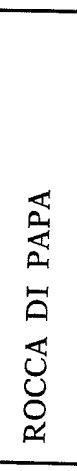 & 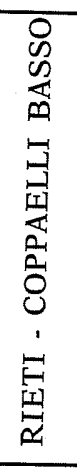 & 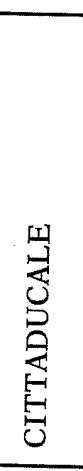 & $\underset{ت}{\mathscr{G}}$ & 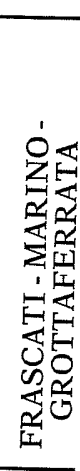 \\
\hline$\stackrel{\Omega}{\sim}$ & 朰 & 贡 & $\stackrel{\sim}{\sim}$ & $\dot{\omega}^{H}$ & $\underline{U}$ & $\underset{-1}{\cup}$ & $\stackrel{\sim}{U}$ & $\circlearrowleft$ & 杂 & Z & 云 & $\underline{U}$ \\
\hline 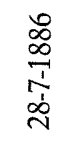 & $\begin{array}{l}\infty \\
\infty \\
\infty \\
\stackrel{1}{1} \\
\stackrel{1}{=}\end{array}$ & $\begin{array}{l}\infty \\
\infty \\
\infty \\
\infty \\
\infty \\
0 \\
0 \\
0\end{array}$ & $\begin{array}{l}\bar{\Omega} \\
\infty \\
\text { ஸ่ } \\
\alpha\end{array}$ & $\begin{array}{l}\vec{a} \\
\stackrel{0}{\vec{d}} \\
\stackrel{1}{1}\end{array}$ & $\begin{array}{l}\stackrel{2}{\infty} \\
\frac{\dot{I}}{\vec{J}} \\
\tilde{N}\end{array}$ & 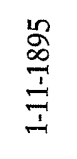 & 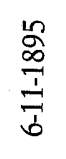 & 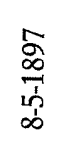 & 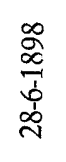 & 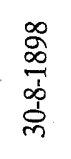 & 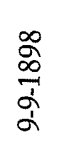 & $\begin{array}{l}\stackrel{2}{2} \\
\frac{1}{1} \\
2 \\
2\end{array}$ \\
\hline$\stackrel{\mathscr{n}}{ \pm}$ & 导 & 导 & $\stackrel{\infty}{+}$ & $\stackrel{g}{ \pm}$ & 음 & 독 & $\stackrel{N}{\sim}$ & in & 茍 & $\underline{n}$ & $\stackrel{n}{n}$ & 12 \\
\hline
\end{tabular}




\begin{tabular}{|c|c|c|c|c|c|c|c|c|c|c|c|}
\hline 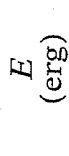 & 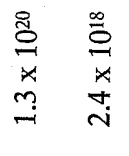 & 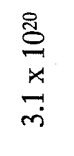 & 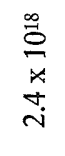 & 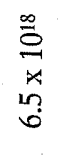 & $\begin{array}{l}\stackrel{\circ}{0} \\
\stackrel{x}{x} \\
\stackrel{y}{y}\end{array}$ & 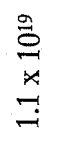 & $\begin{array}{l}\text { 品 } \\
\stackrel{\vec{x}}{x} \\
\stackrel{+}{j}\end{array}$ & 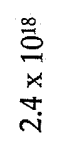 & $\begin{array}{l}\text { 足 } \\
\stackrel{\vec{x}}{*} \\
\stackrel{x}{+}\end{array}$ & 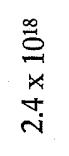 & 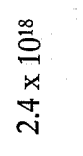 \\
\hline & in $\stackrel{m}{+}$ & है & $\stackrel{m}{q}$ & $\stackrel{8}{8}$ & $\stackrel{\infty}{+}$ & $\stackrel{8}{+}$ & $\stackrel{m}{+}$ & $\stackrel{m}{7}$ & $\stackrel{m}{\partial}$ & $\stackrel{m}{+}$ & $\stackrel{m}{+}$ \\
\hline & in $\Xi$ & \pm .0 & & & & & & & & & $\beth^{\widehat{\theta}}$ \\
\hline & 55 & 岑与 & & 58 & $\stackrel{5}{5}$ & & 5 & 5 & 5 & 5 & \\
\hline
\end{tabular}

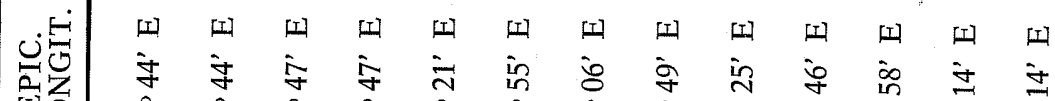

年 守 字 $\bar{y}$ in

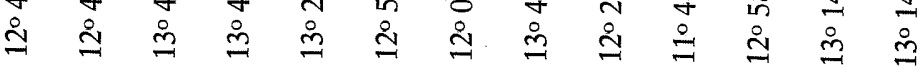

\begin{tabular}{|c|c|c|c|c|c|c|c|c|c|c|c|c|}
\hline 总点 & $\begin{array}{l}Z \\
\text { in } \\
\text { ণे }\end{array}$ & $\begin{array}{l}Z \\
\text { in } \\
\text { in } \\
\text { \&े }\end{array}$ & $\begin{array}{l}Z \\
\dot{y} \\
\dot{J} \\
\stackrel{\circ}{\forall}\end{array}$ & $\begin{array}{l}\text { Z } \\
\dot{J} \\
\stackrel{5}{7} \\
\stackrel{\circ}{7}\end{array}$ & $\begin{array}{l}\text { Z } \\
\dot{Z} \\
\dot{8} \\
\dot{7}\end{array}$ & $\begin{array}{l}Z \\
\dot{Z} \\
\stackrel{\sim}{\circ} \\
\stackrel{y}{+}\end{array}$ & $\begin{array}{l}Z \\
\infty \\
\infty \\
0\end{array}$ & 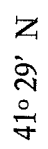 & $\begin{array}{l}z \\
\text { zo } \\
\text { in } \\
\stackrel{0}{y}\end{array}$ & $\begin{array}{l}z \\
\dot{m} \\
\dot{m}\end{array}$ & $\begin{array}{l}Z \\
\dot{+} \\
\stackrel{m}{े} \\
\stackrel{\gamma}{*}\end{array}$ & $\begin{array}{l}Z \\
\stackrel{Y}{Y} \\
\stackrel{y}{\mathcal{y}}\end{array}$ \\
\hline
\end{tabular}

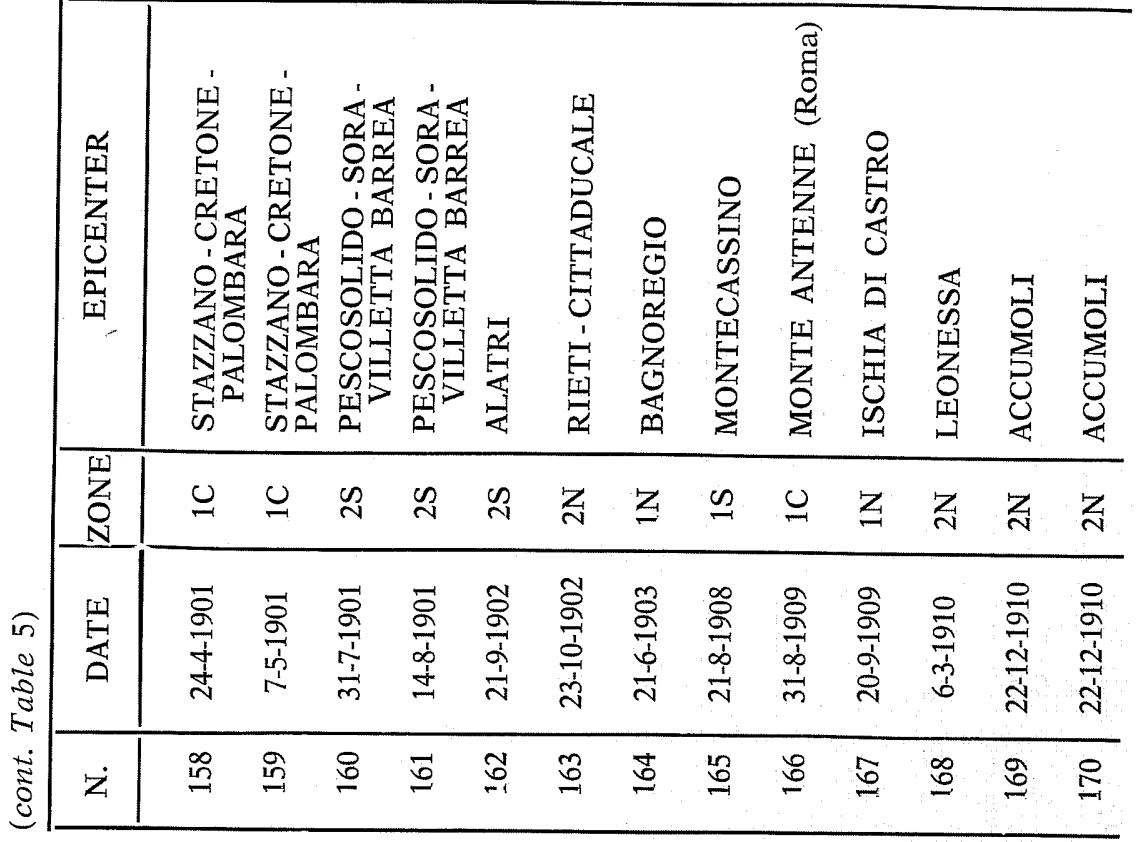




\begin{tabular}{|c|c|c|c|c|c|c|c|c|c|c|c|c|c|c|}
\hline 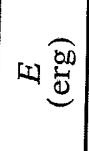 & 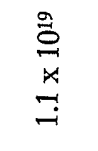 & 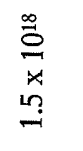 & 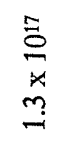 & 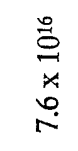 & 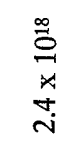 & 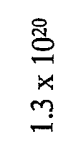 & $\begin{array}{l}\stackrel{\infty}{0} \\
\not<4 \\
\stackrel{+}{v}\end{array}$ & $\begin{array}{l}\stackrel{\infty}{0} \\
\stackrel{\star}{\star} \\
\stackrel{+}{\sim}\end{array}$ & 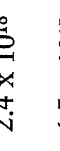 & 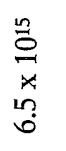 & $\begin{array}{l}\underset{\delta}{0} \\
\stackrel{\dot{m}}{m}\end{array}$ & 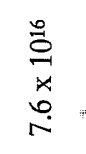 & $\begin{array}{l}\stackrel{\infty}{0} \\
0 \\
i n \\
0 \\
0\end{array}$ & 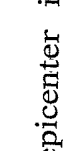 \\
\hline$\Sigma$ & $\stackrel{+}{\mathscr{f}}$ & $\stackrel{\Upsilon}{\forall}$ & 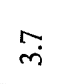 & $\stackrel{\varphi}{\ddot{m}}$ & $\stackrel{\dddot{q}}{*}$ & ت: & $\stackrel{m}{\ddagger}$ & $\stackrel{\sim}{\sigma}$ & $?$ & $\vec{m}$ & m. & $\stackrel{\varphi}{\dot{m}}$ & $\stackrel{n}{\forall}$ & $\Xi$ \\
\hline$\approx \widehat{\Xi}$ & \multicolumn{5}{|c|}{ 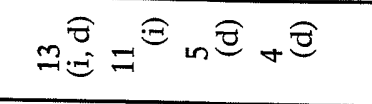 } & \multicolumn{6}{|c|}{ 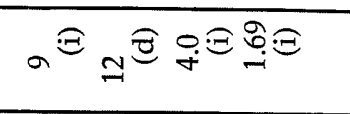 } & \multicolumn{2}{|l|}{$\nabla \widehat{\theta}$} & 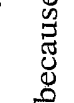 \\
\hline$\stackrel{H}{Z}$ & $\stackrel{5}{5}$ & 5 & 5 & 5 & 5 & $\stackrel{5}{5}$ & 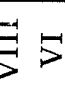 & 5 & & 5 & $\Xi$ & 5 & 今心 & 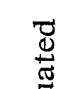 \\
\hline 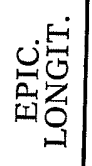 & $\begin{array}{l}\text { 띠 } \\
\dot{\Xi} \\
\stackrel{\circ}{\circ}\end{array}$ & $\begin{array}{l}\text { 되 } \\
\dot{+} \\
\stackrel{+}{ \pm}\end{array}$ & $\begin{array}{l}\text { 디 } \\
\dot{j} \\
\dot{m} \\
\dot{m}\end{array}$ & $\begin{array}{l}\text { 띠 } \\
i ் \\
\text { in } \\
\stackrel{一}{\beth}\end{array}$ & $\begin{array}{l}\text { 더 } \\
\dot{m} \\
\stackrel{m}{\check{I}}\end{array}$ & $\begin{array}{l}\text { 되 } \\
\dot{\delta} \\
\dot{0} \\
\dot{0}\end{array}$ & $\begin{array}{l}\text { II } \\
\text { iे } \\
\text { m } \\
\stackrel{0}{m}\end{array}$ & $\begin{array}{l}\text { II } \\
\grave{O} \\
\stackrel{\Xi}{I}\end{array}$ & $\begin{array}{l}\text { cl } \\
y \\
\text { y } \\
y\end{array}$ & $\begin{array}{l}\text { 디 } \\
\dot{\alpha} \\
\dot{+} \\
\stackrel{0}{7}\end{array}$ & $\begin{array}{l}\text { 디 } \\
\text { in } \\
\text { ஸे } \\
\stackrel{\text { I }}{్}\end{array}$ & $\begin{array}{l}\text { 뙤 } \\
\text { in } \\
\stackrel{n}{0} \\
\stackrel{=}{=}\end{array}$ & $\begin{array}{l}\text { I } \\
i n \\
i n \\
\stackrel{2}{=}\end{array}$ & 岂 \\
\hline 占愳 & $\begin{array}{l}z \\
\grave{y} \\
\stackrel{y}{y} \\
\text { yे }\end{array}$ & $\begin{array}{l}z \\
\dot{z} \\
\circ \\
\dot{y}\end{array}$ & $\begin{array}{l}z \\
\overline{+} \\
\dot{+} \\
\dot{\forall}\end{array}$ & 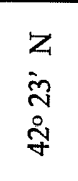 & $\begin{array}{l}Z \\
\dot{8} \\
\stackrel{8}{8}\end{array}$ & 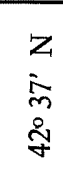 & $\begin{array}{l}Z \\
\text { Z } \\
\stackrel{y}{+} \\
\stackrel{0}{y}\end{array}$ & \begin{tabular}{l}
$z$ \\
ż \\
\multirow{2}{*}{}
\end{tabular} & & $\begin{array}{l}z \\
\dot{Z} \\
\dot{2} \\
\stackrel{0}{+}\end{array}$ & $\begin{array}{l}z \\
\tilde{N} \\
\stackrel{+}{7}\end{array}$ & $\begin{array}{l}\text { Z } \\
\dot{\square} \\
\stackrel{\square}{\stackrel{y}{y}}\end{array}$ & $\begin{array}{l}Z \\
\bar{\forall} \\
\stackrel{+}{+}\end{array}$ & $\begin{array}{l}\approx \\
\frac{y}{0} \\
\frac{0}{n}\end{array}$ \\
\hline 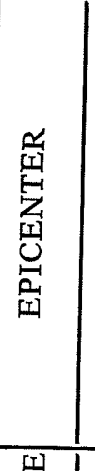 & 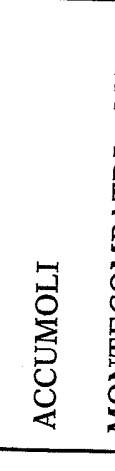 & 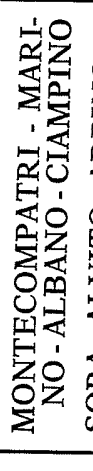 & 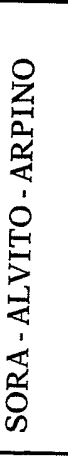 & 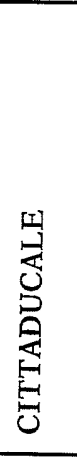 & 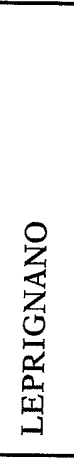 & 究 & 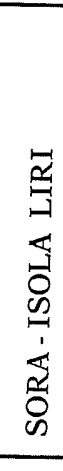 & 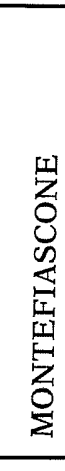 & $\begin{array}{l}0 \\
5 \\
0 \\
5 \\
5 \\
0\end{array}$ & 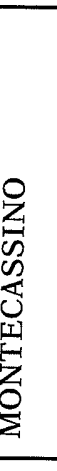 & 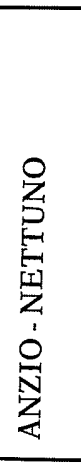 & 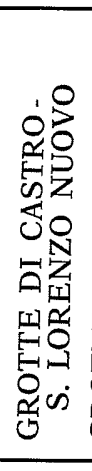 & 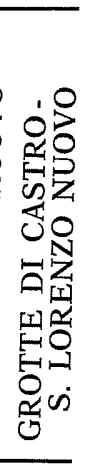 & 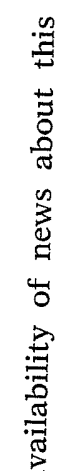 \\
\hline 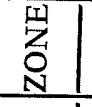 & 惢 & $\circlearrowleft$ & $\stackrel{\sim}{N}$ & Z Z & 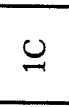 & 云 & $\underset{N}{ }$ & $\underset{\sim}{Z}$ & & $\mathscr{L}^{2}$ & $\mathscr{U}$ & 吕 & 吕 & $\stackrel{乛}{\Xi}$ \\
\hline 装 & $\begin{array}{l}\frac{0}{5} \\
\frac{1}{1} \\
\frac{1}{2}\end{array}$ & $\begin{array}{l}\vec{a} \\
\frac{1}{\dot{z}} \\
\frac{1}{1}\end{array}$ & $\frac{\sqrt{7}}{\frac{1}{1}}$ & $\frac{n}{\frac{n}{\sigma}}$ & $\begin{array}{l}\frac{n}{2} \\
\stackrel{1}{1} \\
\stackrel{i}{n}\end{array}$ & $\frac{0}{\frac{\sigma}{2}}$ & $\begin{array}{l}\frac{5}{2} \\
\frac{1}{a} \\
\stackrel{1}{N}\end{array}$ & 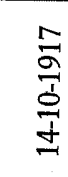 & 5 & $\frac{\bar{a}}{\frac{a}{a}}$ & $\frac{\frac{\sigma}{\sigma}}{\frac{\sigma}{\delta}}$ & $\frac{\vec{\Omega}}{\vec{\Xi}}$ & $\begin{array}{l}\vec{\Xi} \\
\stackrel{\Xi}{I} \\
\beth\end{array}$ & 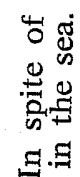 \\
\hline$\ddot{z}$ & $\underline{\Xi}$ & $\underset{-1}{N}$ & $\stackrel{2}{\Xi}$ & $\stackrel{ \pm}{ \pm}$ & $\stackrel{n}{\Xi}$ & 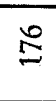 & $\Sigma$ & $\stackrel{\infty}{=}$ & & I & $\underset{\substack{* \\
0}}{*}$ & $\stackrel{\infty}{=}$ & $\stackrel{\infty}{\infty}$ & E. \\
\hline
\end{tabular}




\begin{tabular}{|c|c|c|c|c|c|c|c|c|c|c|c|c|c|}
\hline 山 & 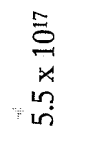 & $\begin{array}{l}\tilde{\sigma} \\
\ddot{x} \\
\dot{\sigma} \\
\dot{m}\end{array}$ & 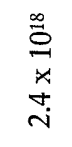 & $\begin{array}{l}\stackrel{\infty}{\circ} \\
\stackrel{1}{*} \\
\stackrel{\sim}{\sim}\end{array}$ & \begin{tabular}{l}
$\infty$ \\
$\stackrel{\infty}{0}$ \\
\\
\multirow{\sim}{*}{}
\end{tabular} & $\begin{array}{c}\infty \\
\stackrel{\infty}{0} \\
\not{x} \\
\stackrel{n}{n} \\
\end{array}$ & 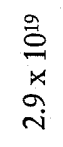 & $\begin{array}{l}\stackrel{\infty}{0} \\
\not{H} \\
\dot{x} \\
\stackrel{+}{v}\end{array}$ & 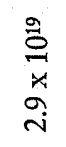 & 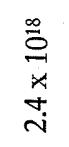 & $\begin{array}{c}n \\
\stackrel{n}{0} \\
x \\
\\
-i\end{array}$ & 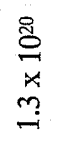 & 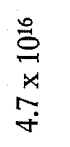 \\
\hline$\Sigma$ & $\stackrel{\circ}{+}$ & ळे & $\stackrel{m}{+}$ & $\stackrel{m}{\rightarrow}$ & $\stackrel{m}{+}$ & $\mathscr{y}$ & $\stackrel{\infty}{\forall}$ & $\stackrel{m}{+}$ & $\stackrel{\infty}{+}$ & $\ddot{q}$ & $\stackrel{\infty}{i}$ & ir: & ֻุ \\
\hline 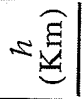 & \multicolumn{3}{|c|}{$\stackrel{0}{\ominus} \Theta$} & & & \multicolumn{2}{|c|}{$=$} & & & & \multicolumn{3}{|c|}{ 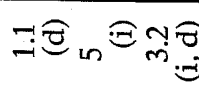 } \\
\hline 吕 & $\stackrel{9}{\supset}$ & 今 & 5 & 5 & 5 & 5 & $\stackrel{5}{5}$ & 5 & $\stackrel{F}{P}$ & 5 & 5 & $\underset{F}{F}$ & 5 \\
\hline 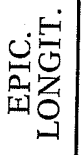 & 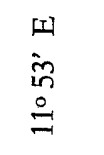 & $\begin{array}{l}\text { 되 } \\
\text { in } \\
\text { in } \\
\stackrel{0}{=}\end{array}$ & 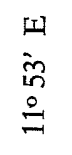 & $\begin{array}{l}\text { 피 } \\
\text { î } \\
\text { ? } \\
\stackrel{0}{=}\end{array}$ & $\begin{array}{l}\text { Iㅓ } \\
\text { in } \\
\text { in } \\
\stackrel{\circ}{=}\end{array}$ & $\begin{array}{l}\text { ㅍ } \\
\dot{\Xi} \\
\stackrel{8}{0}\end{array}$ & $\begin{array}{l}\square \\
\dot{i n} \\
\stackrel{\circ}{=}\end{array}$ & $\begin{array}{l}\text { 되 } \\
\dot{i n} \\
\stackrel{\circ}{=}\end{array}$ & $\begin{array}{l}\text { 띠 } \\
\dot{2} \\
\text { in } \\
\stackrel{0}{=}\end{array}$ & 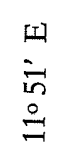 & $\begin{array}{l}\text { Ix } \\
\dot{\text { in }} \\
\stackrel{2}{I}\end{array}$ & $\begin{array}{l}\text { [1 } \\
\text { ஸे } \\
\stackrel{y}{\check{I}}\end{array}$ & $\begin{array}{l}\text { 띠 } \\
\dot{\alpha} \\
\dot{+} \\
\dot{=}\end{array}$ \\
\hline 我葛 & 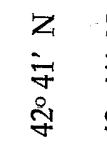 & $\begin{array}{l}Z \\
Z \\
\dot{\forall} \\
\stackrel{+}{7}\end{array}$ & $\begin{array}{l}Z \\
\dot{Z} \\
\stackrel{+}{\square} \\
\stackrel{y}{y}\end{array}$ & \begin{tabular}{l}
$Z$ \\
$Z$ \\
7 \\
\multirow{7}{7}{}
\end{tabular} & $\begin{array}{l}z \\
\bar{y} \\
\dot{+} \\
\dot{y}\end{array}$ & 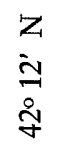 & $\begin{array}{l}Z \\
\text { in } \\
\stackrel{+}{+} \\
\stackrel{+}{y}\end{array}$ & $\begin{array}{l}Z \\
\text { in } \\
\stackrel{+}{\circ} \\
\stackrel{+}{+}\end{array}$ & $\begin{array}{l}Z \\
\text { in } \\
\stackrel{+}{+} \\
\stackrel{+}{y}\end{array}$ & $\begin{array}{l}\text { Z } \\
\text { in } \\
\stackrel{+}{+} \\
\stackrel{y}{4}\end{array}$ & $\begin{array}{l}\text { Z } \\
\dot{m} \\
\dot{y} \\
\text { ஸे }\end{array}$ & $\begin{array}{l}z \\
\dot{z} \\
\dot{y} \\
\dot{y}\end{array}$ & $\begin{array}{l}Z \\
Z \\
\ddot{+} \\
\stackrel{+}{y}\end{array}$ \\
\hline 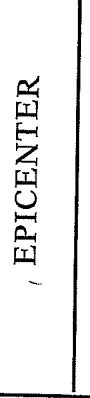 & 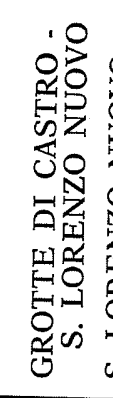 & 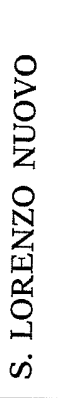 & 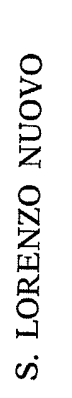 & 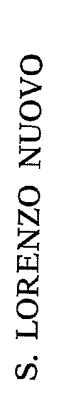 & 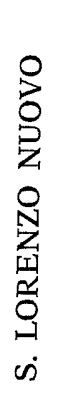 & 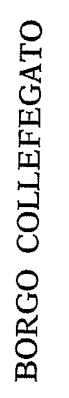 & 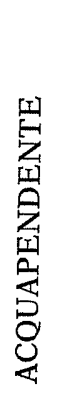 & 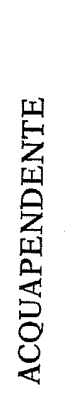 & 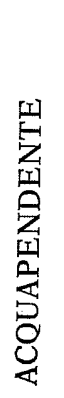 & 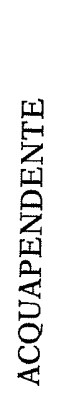 & 丞 & $\sum_{\substack{1 \\
Z}}^{E}$ & $\sum_{0}^{0}$ \\
\hline 甹 & Z & 杰 & ZZ & 杰 & Z & Z & 烃 & $Z_{1}$ & Z & Z & Z & $\bigcup$ & 艺 \\
\hline 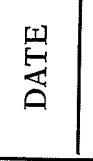 & $\frac{\vec{\alpha}}{\stackrel{\Xi}{ \pm}}$ & $\begin{array}{l}\widetilde{\Xi} \\
\frac{1}{2} \\
\frac{\sqrt{N}}{N}\end{array}$ & $\frac{\widetilde{Z}}{\frac{\sigma}{\sigma}}$ & $\frac{\widetilde{N}}{\stackrel{\sim}{N}}$ & $\frac{\widetilde{N}}{\frac{\tilde{\sigma}}{\vec{d}}}$ & $\begin{array}{l}\underset{\sigma}{\sigma} \\
\stackrel{n}{n}\end{array}$ & 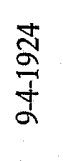 & 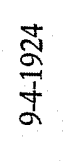 & 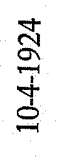 & 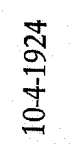 & 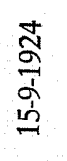 & 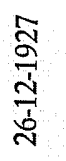 & 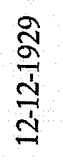 \\
\hline$z$ & $\mathscr{\infty}$ & サ & $\stackrel{\mathscr{L}}{\infty}$ & $\stackrel{\circ}{\cong}$ & $\underset{-1}{\infty}$ & $\stackrel{\infty}{\infty}$ & 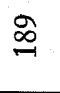 & 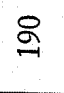 & $\vec{\sigma}$ & $\widetilde{\sigma}$ & $\approx$ & $\stackrel{\Xi}{\Xi}$ & $\stackrel{2}{\sigma}$ \\
\hline
\end{tabular}




\begin{tabular}{|c|c|c|c|c|c|c|c|c|c|c|c|c|c|c|c|}
\hline 되 & $\begin{array}{l}\stackrel{0}{0} \\
\stackrel{1}{x} \\
\stackrel{-1}{-}\end{array}$ & 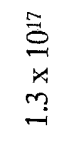 & 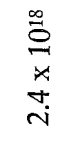 & 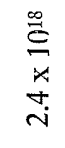 & $\underset{a}{a}$ & 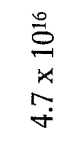 & 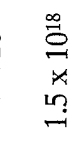 & & $\begin{array}{l}\stackrel{D}{d} \\
\dot{x} \\
\stackrel{i}{i}\end{array}$ & $\begin{array}{l}\stackrel{\infty}{\sigma} \\
x \\
1 n \\
0\end{array}$ & 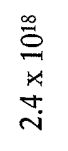 & $\begin{array}{l}5 \\
a \\
a\end{array}$ & $\begin{array}{l}\stackrel{\infty}{0} \\
\not{x} \\
n \\
0 \\
0\end{array}$ & $\begin{array}{l}\stackrel{\infty}{\sigma} \\
\underset{x}{\not} \\
\stackrel{+}{\dot{*}}\end{array}$ & $\begin{array}{l}\tilde{a} \\
\dot{0} \\
\not{x} \\
\ddot{a}\end{array}$ \\
\hline$\Sigma$ & $\underset{\sim}{\sim}$ & $\dot{m}$ & $\ddot{q}$ & $\ddot{q}$ & $\stackrel{\infty}{\sim}$ & $\stackrel{n}{m}$ & & $\underset{+}{\stackrel{y}{*}}$ & in & $\stackrel{\ln }{+}$ & $\ddot{q}$ & $\stackrel{*}{7}$ & $\stackrel{\text { In }}{+}$ & $\stackrel{+}{+}$ & $\stackrel{*}{*}$ \\
\hline 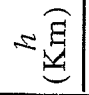 & \multicolumn{2}{|c|}{$\sim \widehat{0}$} & & & \multicolumn{4}{|c|}{ 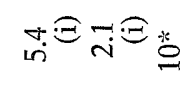 } & \multicolumn{2}{|c|}{$2 \ominus$} & & \multicolumn{2}{|c|}{ 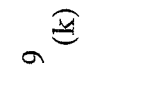 } & \multicolumn{2}{|c|}{$\sin _{a} \cong$} \\
\hline 荣 & $\$$ & 5 & 5 & 5 & 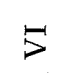 & & $\mathscr{5}$ & & 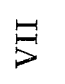 & $\stackrel{5}{5}$ & 15 & 5 & $\stackrel{5}{5}$ & 5 & 5 \\
\hline 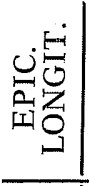 & 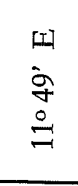 & $\begin{array}{l}\text { ㅍ } \\
\dot{\sigma} \\
\circ \\
\stackrel{ }{\beth}\end{array}$ & $\begin{array}{l}\text { 뙤 } \\
\dot{\alpha} \\
\stackrel{+}{二}\end{array}$ & 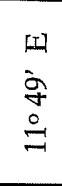 & $\begin{array}{l}\text { II } \\
\text { 혀 } \\
\stackrel{0}{7}\end{array}$ & 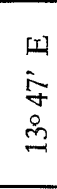 & & 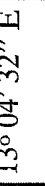 & $\begin{array}{l}\text { ㅍ } \\
\text { in } \\
\text { ○े } \\
\stackrel{y}{y}\end{array}$ & \begin{tabular}{l} 
ㅍ \\
in \\
\multirow{+}{0}{} \\
$\stackrel{y}{y}$
\end{tabular} & $\begin{array}{l}\text { 되 } \\
\dot{\infty} \\
= \\
\stackrel{0}{=}\end{array}$ & $\begin{array}{l}\text { II } \\
\dot{O} \\
0 \\
\stackrel{0}{=}\end{array}$ & $\begin{array}{l}\text { 띠 } \\
\text { ĩ } \\
\stackrel{0}{=}\end{array}$ & 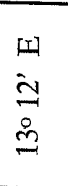 & \begin{tabular}{l} 
디 \\
in \\
0 \\
0 \\
\hdashline
\end{tabular} \\
\hline 岕点 & 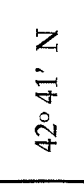 & 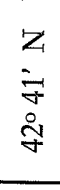 & $\begin{array}{l}Z \\
Z \\
\overrightarrow{7} \\
\stackrel{+}{7}\end{array}$ & 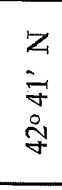 & $\begin{array}{l}Z \\
\dot{y} \\
\dot{y} \\
\dot{y}\end{array}$ & $\begin{array}{l}Z \\
\dot{m} \\
\dot{g} \\
\dot{\nabla}\end{array}$ & 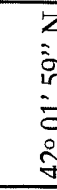 & & 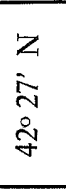 & 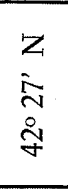 & $\begin{array}{l}\text { Z } \\
\stackrel{y}{y} \\
\stackrel{\text { ๖े }}{y}\end{array}$ & $\begin{array}{l}Z \\
\stackrel{Z}{Z} \\
\stackrel{\sim}{+}\end{array}$ & $\begin{array}{l}Z \\
\dot{3} \\
\dot{+} \\
\stackrel{5}{+}\end{array}$ & 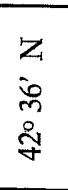 & $\begin{array}{l}z \\
\dot{8} \\
\stackrel{8}{y} \\
\stackrel{y}{+}\end{array}$ \\
\hline 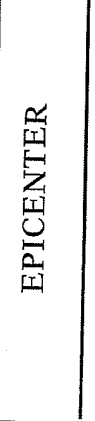 & 是 & $\begin{array}{l}0 \\
Z \\
Z \\
\vdots \\
0\end{array}$ & 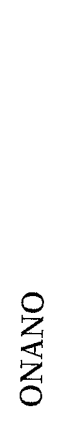 & \begin{tabular}{l}
0 \\
\multirow{Z}{Z}{} \\
$\vdots$ \\
0
\end{tabular} & 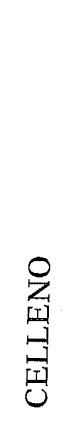 & 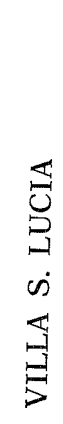 & 要 & $\frac{\mathbb{2}}{2}$ & 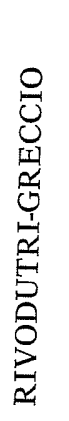 & 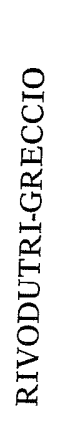 & 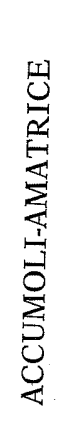 & & 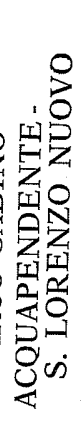 & 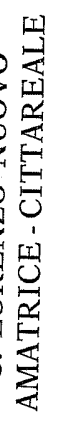 & 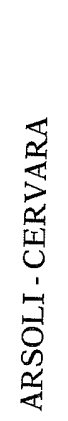 \\
\hline $\begin{array}{l}\text { II } \\
\text { 苍 } \\
\text { N } \\
\end{array}$ & 羊 & 吕 & Z & 旮 & Z & $\stackrel{5}{\rightarrow}$ & $\stackrel{n}{\sim}$ & 3 & Z & $Z_{i}$ & 㤂 & Z & 写 & 㤂 & $\stackrel{\sim}{\sim}$ \\
\hline 壱 & 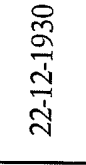 & 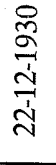 & 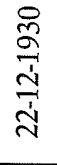 & 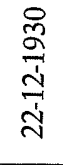 & $\begin{array}{l}\tilde{n} \\
\frac{1}{\omega} \\
\stackrel{n}{\sigma}\end{array}$ & 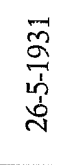 & $\frac{7}{\bar{Z}}$ & $\frac{1}{5}$ & 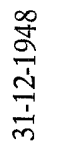 & $\frac{\frac{9}{2}}{\frac{1}{4}}$ & $\begin{array}{l}\stackrel{ }{2} \\
\stackrel{1}{a} \\
\stackrel{2}{\sim}\end{array}$ & $\begin{array}{l}\hat{2} \\
\stackrel{2}{+} \\
\stackrel{1}{=}\end{array}$ & 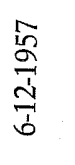 & $\frac{\mathscr{D}}{5}$ & 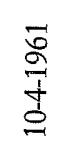 \\
\hline$z$ & 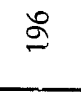 & 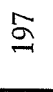 & $\stackrel{\infty}{\stackrel{2}{2}}$ & g & §্ণ & $\tilde{\nabla}$ & $\S$ & & $\stackrel{\sim}{\sim}$ & 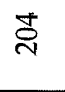 & $\stackrel{n}{\stackrel{\sim}{N}}$ & $\stackrel{ }{~}$ & ิㅗ & $\stackrel{\infty}{\stackrel{\circ}{N}}$ & 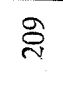 \\
\hline
\end{tabular}


(cont. Table 5)

\begin{tabular}{|c|c|c|c|c|c|c|c|c|c|}
\hline N. & DATE & ZONE & EPICENTER & $\begin{array}{l}\text { EPIC. } \\
\text { LATIT. }\end{array}$ & $\begin{array}{l}\text { EPIC. } \\
\text { LONGIT. }\end{array}$ & INT & $\begin{array}{c}h \\
(\mathrm{Km})\end{array}$ & $M$ & $\begin{array}{c}E \\
(\operatorname{erg})\end{array}$ \\
\hline 210 & $31-10-1961$ & $2 \mathrm{~N}$ & $\begin{array}{l}\text { ANTRODOCO - BORGO } \\
\text { VELINO - COLLE RINALDO }\end{array}$ & $42^{\circ} 21^{\prime} 20.4^{\prime \prime} \mathrm{N}$ & $13 \circ 01^{\prime} 05.5^{\prime \prime} \mathrm{E}$ & VIII & $6^{*}$ & $5.18^{*}$ & $1.9 \times 10^{20}$ \\
\hline 211 & $2-2-1963$ & $2 \mathrm{~N}$ & CITTADUCALE & $42^{\circ} 24^{\prime} \mathrm{N}$ & $12^{\circ} 54^{\prime} \mathrm{E}$ & VIII & $\begin{array}{l}5 \\
(\mathrm{k})\end{array}$ & $5.1^{*}$ & $1.3 \times 10^{20}$ \\
\hline 212 & $21-7-1963$ & $2 \mathrm{~N}$ & ARAFRANCA & $42^{\circ} 36^{\prime} \mathrm{N}$ & $13^{\circ} 18^{\prime} \mathrm{E}$ & VII & 11 & $4.9^{*}$ & $4.7 \times 10^{19}$ \\
\hline 213 & $24-7-1963$ & $2 \mathrm{~N}$ & ARAFRANCA & $42^{\circ} 36^{\prime} \mathrm{N}$ & $13^{\circ} 18^{\prime} \mathrm{E}$ & VI & 15 & $4.4^{*}$ & $4.0 \times 10^{18}$ \\
\hline 214 & $24-7-1963$ & $2 \mathrm{~N}$ & ARAFRANCA & $42^{\circ} 36^{\prime} \mathrm{N}$ & 13० $18^{\prime} \mathrm{E}$ & VI & 13 & $4.3^{*}$ & $2.4 \times 10^{18}$ \\
\hline 215 & $2-9-1963$ & $1 \mathrm{~N}$ & VALLERANO-VIGNANELLO & $42^{\circ} 22^{\prime} \mathrm{N}$ & $12^{\circ} 15^{\prime} \mathrm{E}$ & VI & 3 & $3.5^{*}$ & $4.7 \times 10^{16}$ \\
\hline 216 & $16-10-1966$ & $1 \mathrm{~S}$ & ARPINO - SANTOPADRE & $41^{\circ} 36^{\prime} \mathrm{N}$ & $13 \circ 36^{\prime} \mathrm{E}$ & VI & 6 & 3.8 & $2.1 \times 10^{17}$ \\
\hline 217 & $4-1-1968$ & $1 \mathrm{~N}$ & COLLEVECCHIO - TARANO & $42^{\circ} 24^{\prime} \mathrm{N}$ & $12^{\circ} 30^{\prime} \mathrm{E}$ & VI & $\begin{array}{l}5 \\
(\mathrm{k})\end{array}$ & $3.7^{*}$ & $1.3 \times 10^{17}$ \\
\hline 218 & $17-4-1969$ & $2 S$ & TERELLE - CASSINO & $41^{\circ} 36, \mathrm{~N}$ & $13^{\circ} 48^{\prime} \mathrm{E}$ & $\begin{array}{l}\text { VII- } \\
\text { VIII }\end{array}$ & $6^{*}$ & $4.6^{*}$ & $1.1 \times 10^{19}$ \\
\hline 219 & $22-11-1970$ & $2 \mathrm{~S}$ & S. VITTORE & $41^{\circ} 32^{\prime} \mathrm{N}$ & $13^{\circ} 56, \mathrm{E}$ & VI & $6^{*}$ & 3.8 & $2.1 \times 10^{17}$ \\
\hline 220 & $5-2-1971$ & $1 \mathrm{~N}$ & TUSCANIA & $42^{\circ} 25^{\prime} 07^{\prime \prime} \mathrm{N}$ & $11^{\circ} 52^{\prime} 43.6^{\prime \prime} \mathrm{E}$ & $\begin{array}{l}\text { VII- } \\
\text { VIII }\end{array}$ & $2^{*}$ & $4.5^{*}$ & $6.5 \times 10^{18}$ \\
\hline 221 & $30-10-1973$ & $2 S$ & VALLEROTONDA & $41^{\circ} 35^{\prime} \mathrm{N}$ & $13^{\circ} 57^{\prime} \mathrm{E}$ & VI & 13 & $4.3^{*}$ & $2.4 \times 10^{18}$ \\
\hline 222 & $5-11-1973$ & $2 \mathrm{~S}$ & ATINA - FROSINONE & $41^{\circ} 41^{\prime} \mathrm{N}$ & $13^{\circ} 48^{\prime} \mathrm{E}$ & $\begin{array}{l}\text { VI- } \\
\text { VII }\end{array}$ & 5 & $4^{*}$ & $5.5 \times 10^{17}$ \\
\hline 223 & $5-11-1973$ & $2 S$ & ATINA - FROSINONE & $41^{\circ} 41^{\prime} \mathrm{N}$ & $13^{\circ} 48^{\prime} \mathrm{E}$ & VI & 7 & $4.6 *$ & $1.1 \times 10^{19}$ \\
\hline
\end{tabular}




\section{REFERENCES}

Agamennone G., 1911 - Il terremoto laziale del 10 aprile 1911. Rend. R. Acc. N. Lincei Ser. 5a, XX, p. 12.

Agamennone G., 1933 - Considerazioni sopra gli ipocentri sismici dei Colli Laziali. Rend. R. Acc. N. Lincei, Ser, 6a, XVII, p. 725.

Baratta M., 1901 - I terremoti d'Italia. Fr. Bocca, Torino.

BCIS: Bureau Central International Séismologique - Strasbourg.

BENIOFF H., 1951 - Global Strain Sequence of Great Shallow Earthquakes. "Bull. Geol. Soc. Am.", 62.

Biagi P.F., Corbò G., Spadea M.C., 1974 - Evoluzione dei fenomeni sismici nell'Appennino centrale. "Annali di Geofisica", XXVII, 34, p. 539.

Bollettino Sismico Mensile, ING, Roma.

Calor P., 1942 - Attività sismica in Italia nel decennio 1930-1939. Le Monnier, Firenze.

Carozzo M.T., de Visintini G., Giorgetti F., Iaccarino E., 1973 - General Catalogue of Italian Earthquakes. CNEN, RT/PROT, p. 12.

Cavasino A., 1935 - I terremoti d'Italia nel trentacinquennio 1899-1933. Ist. Poligrafico dello Stato, Roma.

Console R., Sonaglia, A., 1972 - Studio del terremoto di Tuscania. "Annali di Geofisica », XXV, 3, p. 367.

De Panfilss M., 1959 - Attività sismica in Italia dal 1953 al 1957. "Annali di Geofisica », XII, 1, p. 21.

Di Filippo D., 1942 - Il terremoto di Cervara di Roma dell'8 settembre 1941. "Boll. Soc. Sismol. Italiana », XL, 1-2, p. 3.

Di Filippo D., Marcelli L., 1950 - Magnitudo ed energia dei terremoti in Italia. "Annali di Geofisica ", III ,3, p. 337.

Gasparini C., Giovani L., 1973 - Attività sismica in Italia nell'anno 1970. "Annali di Geofisica", XXVI, 2-3, p. 445.

Gutenberg B., Richter, C. F., 1942 - Earthquake Magnitude, Intensity, Energy and Acceleration. "Bull. Seismol. Soc. Amer, », 32, p. 163-191. 
Gutenberg B., Richter C.F., 1944 - Frequency of earthquakes in California. "Bull. Seismol. Soc. Amer. », 34, p. 185-188.

Gutenberg B., Richter C.F., 1954 - Seismicità of Earth and Associated Phenomena. Princeton University Press (second edition).

IAcCaRINo E., 1973 - Sismicità dell'Italia nei secoli scorsi. CNEN RT/ PROT, 7.

Kárník V., 1969 - Seismicità of the European Area. Part I. Czechoslovak Academy of Sciences.

Marcelli L., Montecchi A., 1962 - Contributi per uno studio sulla sismicità dell'Italia. "Annali di Geofisica ", XV, 2-3, p. 159.

Marcelli L., Pannocchia G., 1962 - L'attività sismica nella zona delle sorgenti del Peschiera. (Parte I). "Annali di Geofisica ", XV, 4, p. 301.

Marcelli L., Pannocchia, 1963 - L'at tività sismica nella zona delle sorgenti del Peschiera. (Parte II). "Annali di Geofisica ", XVI, 4, p. 645.

USCGS: U.S. Coast and Geodetic Survey - Washington. 